\title{
Large Eddy Simulation of the transition from sheet to cloud cavitation over a wedge
}

\author{
Aswin Gnanaskandan ${ }^{1}$, Krishnan Mahesh ${ }^{2, *}$ \\ Aerospace Engineering and Mechanics, University of Minnesota
}

\begin{abstract}
LES of sheet to cloud cavitation over a wedge is performed at Reynolds number $R e=$ 200,000 (based on the wedge height and free stream velocity) and cavitation number $\sigma=2.1$. The attached sheet cavity grows upto a critical length, after which it breaks into a cloud cavity which is highly three-dimensional and vortical in nature. The mean and RMS void fraction profiles obtained inside the cavity are compared to experiment and good agreement is observed. The frequency of the shedding process is obtained from point spectra at several locations and the obtained frequency is found to agree with the experiment. It is observed that the mean pressure at the wedge apex does not fall below vapor pressure; however cavitation occurs there due to the unsteady pressure falling below vapor pressure. The maximum mean void fraction occurs in the sheet cavity and is about 0.5, while the cloud region has even lesser amount of void fraction. The velocity fluctuations immediately downstream of the cavity show comparable streamwise and spanwise values, while the spanwise values are smaller in comparison inside the cavity region. The probability density function of void fraction examined at several locations inside the cavity show that the mean value obtained from time averaged data is very different from the most probable value of void fraction, indicating the considerable unsteadiness of the flow. The pressure waves produced upon cloud collapse are found to display both cyclic behavior and small scale transient behavior downstream of the wedge. The LES results agree better with experiment than unsteady RANS in predicting this highly unsteady flow.
\end{abstract}

Keywords: Sheet to cloud cavitation, Re-entering jet, Large Eddy Simulation, Cavity auto-oscillation.

\section{Introduction}

Cavitation refers to the formation of vapor when pressure in a liquid drops below vapor pressure. It occurs in a wide variety of situations such as valves, orifices and propulsor blades. The formation of vapor is often followed by a growth of the vapor cavity and its violent collapse under high pressure gradient. The physical consequences of this collapse include noise, vibration and surface erosion. Sheet cavitation and its transition to cloud cavitation is of great practical interest since the highly unsteady flow

\footnotetext{
* Corresponding author

Email addresses: gnan0009@aem.umn.edu (Aswin Gnanaskandan), kmahesh@aem.umn.edu (Krishnan Mahesh)

${ }^{1}$ Graduate Student

${ }^{2}$ Professor
} 


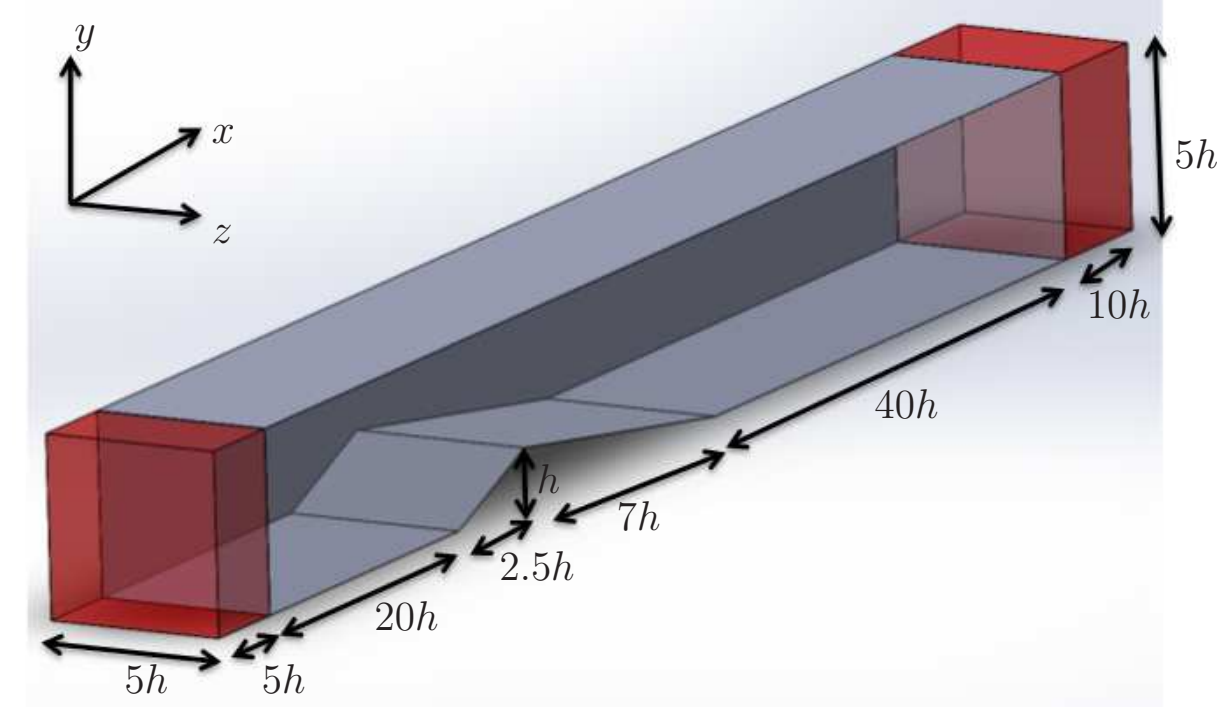

Figure 1: Schematic of the computational domain (Not to scale).

can induce significant fluctuations in the thrust and torque of marine propellers. The collapse of the cloud also causes material damage to the blades. Several experiments have been conducted to understand the sheet to cloud transition mechanism. Arndt et al. [2] studied a NACA 0015 hydrofoil and observed two types of behavior based on the parameter $\frac{\sigma}{2 \alpha}$, where $\sigma$ is the cavitation number and $\alpha$ is the angle of attack. They concluded that a re-entering jet was responsible for cavity destabilization for $\frac{\sigma}{2 \alpha}>4$, while at lower values, a bubbly shock dominates the flow. More recently, Ganesh [13] performed experiments on a wedge and also observed that a propagating shock wave is dominant at lower cavitation numbers; they term this regime 'periodic cavitation'. At slightly larger cavitation numbers, they observe intermittent cloud shedding and term this 'transitory cavitation'. In this regime, they observe that re-entering jet is dominant during earlier stages and a propagating shock wave is dominant during later stages of the experimental cycle. Leroux et al. [27] observed a quasi stable partial sheet cavity on a hydrofoil for cavity lengths less than half the chord length and sheet to cloud cavitation for lengths greater than half the chord length. They also agreed with the conclusions of Arndt et al. [2] but modified the parameter to be $\frac{\sigma}{2\left(\alpha-\alpha_{0}\right)}$, where $\alpha_{0}$ is zero for symmetric hydrofoils. Stutz and Reboud [37] measured local void fraction inside the cavity formed on a wedge and confirmed the presence of a re-entering jet along the wall. Experiments by Pham et al. [29] also showed the role of re entering jet in cloud formation on a hydrofoil. Kawanami et al. [21] showed that a small obstacle in the path of the re -entering jet was able to prevent the formation of cloud cavity, demonstrating the role of re-entering jet in producing the cloud. Interestingly though, Ganesh [13] also placed an obstacle inside the cavity and showed that it did not prevent the formation of a cloud cavity, consistent 


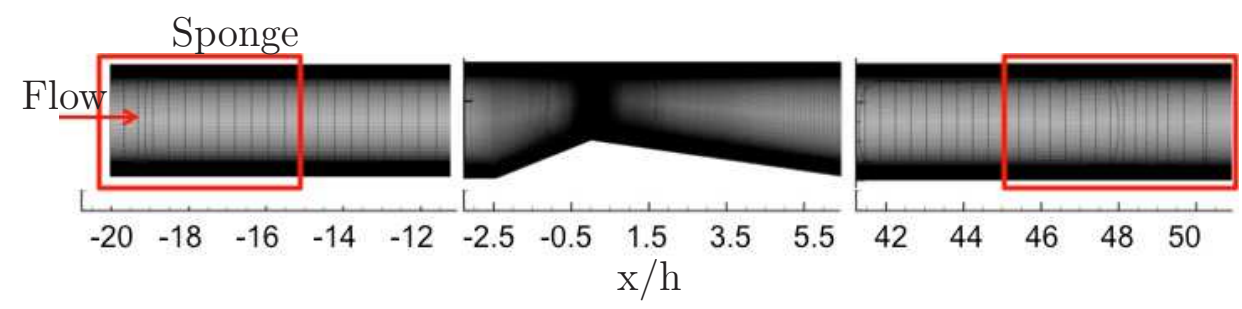

Figure 2: $2 D$ slice of the computational domain showing the mesh.

with the existence of a bubbly shock which exists not only close to the wall but spans the cavity thickness. Foeth et al. [11] observed a re-entrant flow ('side re-entrant jet') from the sides of the cavity towards the center plane colliding in the closure region of the cavity and pinching off a part of the sheet resulting in periodic shedding. Callenaere et al. [5] established the importance of an adverse pressure gradient at the cavity closure for the formation of a re-entering jet. They observed two types of cavity, thick and thin based on the amount of interaction between the re-entering jet and the cavity interface. For thick cavities, the interaction between the re-entering jet and the cavity is minimum until the re-entering jet reaches the leading edge of the cavity, giving rise to the classical sheet to cloud transition. On the other hand in a thin cavity, the interaction with re-entering jet causes the cavity to split into many small structures. In this case, although the cavity does not auto-oscillate, the re-entering jet is still periodic with a Strouhal number in the range 0.2 - 0.4. Laberteaux and Ceccio [26] further classified cavities as open and closed based on the absence and presence of re-entering jet respectively. A closed cavity has a clear interface and a re-entering jet is often found, whereas an open cavity is typically frothy with no clear re-entering jet.

Most computational studies of sheet to cloud cavitation have employed the homogeneous mixture model [24] where the multiphase fluid mixture is treated as a single compressible fluid. The major difference between the several studies using this model lies in the equation of state of the mixture, and the mass transfer model that governs the rate of evaporation and condensation. A comparative study of various mass transfer models can be found in $[12,33]$. Another major difference among these studies is whether they use pressure based or density based algorithms both of which have to address the mixed incompressible -compressible nature of cavitating flows. The pressure based algorithms proposed for single phase flows often experience convergence problems in cavitating flows. This is primarily because of the compressible nature of cavitating flows. The main challenge in these algorithms is to obtain the correct speed of sound and various modifications to pressure based algorithms have been proposed [4, 32, 35] to address this issue. Density based algorithm solves the compressible Navier-Stokes equations. Here, convergence issues arise due to the nearly incompressible nature of the liquid. To alleviate this, preconditioning has been suggested as a viable technique [25, 1, 10]. However, preconditioning affects the time accuracy of the computations and almost always has to be complemented with dual time stepping where the preconditioner is applied in the pseudo time level.

A number of studies have used the homogeneous mixture assumption to study cavitation over two-dimensional geometries (e.g. [35, 8, 34, 16, 22]), and three-dimensional geometries with variation in the span and sidewalls (e.g. [30, 31, 23, 19]). Most of the above studies used unsteady RANS to model the turbulence. Although Hoekstra et al. [17] have shown a RANS model to predict unsteady shedding without any ad-hoc corrections, most RANS models need an ad-hoc suppression of eddy viscosity at the cavity interface in order to predict sheet to cloud cavitation (see e.g. [8]). Recently, LES has 


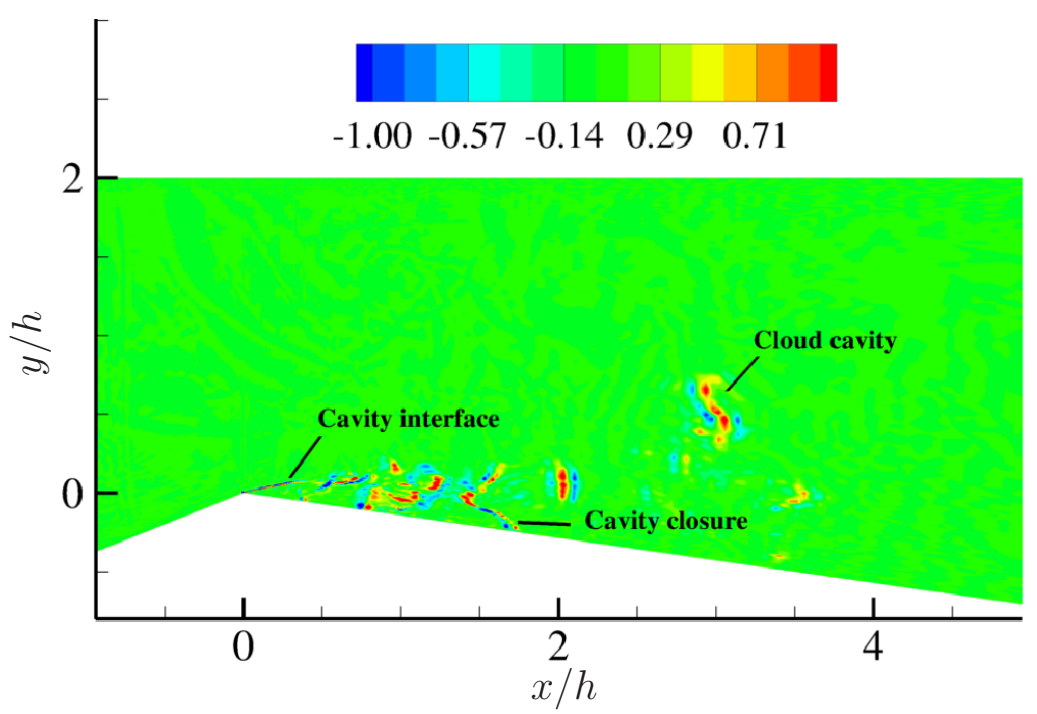

Figure 3: Dissipative flux showing localization near cavity interface, closure and inside cloud cavity.

been considered since it can predict flow unsteadiness better without ad-hoc modifications (e.g. [15, 4, 9, 20, 38]). The various LES studies differ in their detail. Bensow and Bark [4] used an incompressible segregated method using a pressure Poisson equation to predict cavitation over a hydrofoil and concluded that the discrepancy in the cavity length predicted by their method could be a result of the incompressible method. Dittakavi et al. [9] used a fully compressible Favre-filtered algorithm for simulating cavitating flow over a wedge; however they artificially modified the sound speed to reduce the stiffness of the system. This method predicts the general dynamics of sheet to cloud cavitation well, however the frequencies predicted are not accurate. Huang et al. [18] also used a compressible method to simulate sheet to cloud cavitation over a hydrofoil and found that their method over predicts the vapor volume fraction inside the cavity and this contributed to the discrepancy in the velocity and vorticity field. Arndt et al. [3] used a weakly compressible approach to perform LES of sheet to cloud cavitation over a NACA hydrofoil and studied the large scale structures produced in the wake of the hydrofoil. However, they specify an arbitrary Mach number in the liquid to accelerate their computation. They justify this in their study by saying that their primary interest is to study hydrodynamic quantities and not acoustic quantities. Yu et al. [40] used a $k-\mu$ SGS model to perform LES of sheet to cloud cavitation over a hydrofoil and obtained good comparison for the re-entering jet velocity. However the cavity length predicted by their method differs significantly from the experimental values. The studies of Wang and Ostoja-Starzewski [38] and Ji et al. [20] also used compressible LES to study cavitation over hydrofoil. Few past LES studies have shown satisfactory quantitative comparisons for void fraction. In this study, we quantitatively compare the length of the cavity, Strouhal number corresponding to cavity auto-oscillation and void fraction profiles inside the cavity with the experiments of Ganesh [13].

The paper is organized into the following sections. Section 2 explains the governing equations, physical model and the numerical method used. Section 3 describes the problem setup, mesh and boundary conditions. Section 4 discusses various aspects of the sheet to cloud transition process. The time evolution of sheet to cloud transition, the role of re-entering jet and pressure waves are discussed in detail. Comparison of both LES and unsteady RANS results to experiment is also discussed. A brief summary is then provided in Section 5. 


\section{Governing equations and numerical method}

The homogeneous mixture model assumes the mixture of constituent phases to be a single compressible fluid and the phases to be in thermal and mechanical equilibrium. Surface tension effects are assumed small and are neglected. The governing equations are the compressible Navier Stokes equation for the mixture of liquid and vapor along with a transport equation for vapor. The governing equations are Favre averaged and then spatially filtered to perform LES. A dynamic Smagorinsky model is used for the subgrid terms. The unfiltered governing equations are:

$$
\begin{aligned}
\frac{\partial \rho}{\partial t} & =-\frac{\partial}{\partial x_{k}}\left(\rho u_{k}\right) \\
\frac{\partial \rho u_{i}}{\partial t} & =-\frac{\partial}{\partial x_{k}}\left(\rho u_{i} u_{k}+p \delta_{i k}-\sigma_{i k}\right) \\
\frac{\partial \rho e_{s}}{\partial t} & =-\frac{\partial}{\partial x_{k}}\left(\rho e_{s} u_{k}-Q_{k}\right)-p \frac{\partial u_{k}}{\partial x_{k}}+\sigma_{i k} \frac{\partial u_{i}}{\partial x_{k}} \\
\frac{\partial \rho Y}{\partial t} & =-\frac{\partial}{\partial x_{k}}\left(\rho Y u_{k}\right)+S_{e}-S_{c}
\end{aligned}
$$

where $\rho, u_{i}, e_{s}$ and $p$ are density, velocity, internal energy, and pressure respectively, of the mixture and $Y$ is the vapor mass fraction. The mixture density

$$
\rho=\rho_{l}(1-\alpha)+\rho_{g} \alpha,
$$

where $\rho_{l}$ is the density of liquid and $\rho_{g}$ is the density of vapor. $\alpha$ is the vapor volume fraction which is related to the vapor mass fraction by

$$
\rho_{l}(1-\alpha)=\rho(1-Y) \text { and } \rho_{g} \alpha=\rho Y .
$$

The system is closed using a mixture equation of state $([15,34])$ :

$$
p=Y \rho R_{g} T+(1-Y) \rho K_{l} T \frac{p}{p+P_{c}} .
$$

Here, $R_{g}=461.6 \mathrm{~J} / \mathrm{KgK}, K_{l}=2684.075 \mathrm{~J} / \mathrm{KgK}$ and $P_{c}=786.333 \times 10^{6} \mathrm{~Pa}$ are constants associated with the equation of state of vapor and liquid. The expression for $e_{s}$ is given by

$$
\rho e_{s}=\rho C_{v m} T+\rho(1-Y) \frac{P_{c} K_{l} T}{p+P_{c}}
$$

where $C_{v m}=(1-Y) C_{v l}+Y C_{v g}$ and $C_{v l}$ and $C_{v g}$ are the specific heats at constant volume for liquid and vapor respectively. The viscous stress $\sigma_{i j}$ and heat flux $Q_{i}$ are given by

$$
\begin{aligned}
\sigma_{i j} & =\mu\left(\frac{\partial u_{i}}{\partial x_{j}}+\frac{\partial u_{j}}{\partial x_{i}}-\frac{2}{3} \frac{\partial u_{k}}{\partial x_{k}} \delta_{i j}\right) \text { and } \\
Q_{i} & =k \frac{\partial T}{\partial x_{i}}
\end{aligned}
$$

where the $\mu$ and $k$ are mixture viscosity and mixture thermal conductivity respectively. $S_{e}$ and $S_{c}$ are source terms for evaporation of water and condensation of vapor and are given by

$$
\begin{aligned}
& S_{e}=C_{e} \alpha^{2}(1-\alpha)^{2} \frac{\rho_{l}}{\rho_{g}} \frac{\max \left(p_{v}-p, 0\right)}{\sqrt{2 \pi R_{g} T}} \\
& S_{c}=C_{c} \alpha^{2}(1-\alpha)^{2} \frac{\max \left(p-p_{v}, 0\right)}{\sqrt{2 \pi R_{g} T}}
\end{aligned}
$$




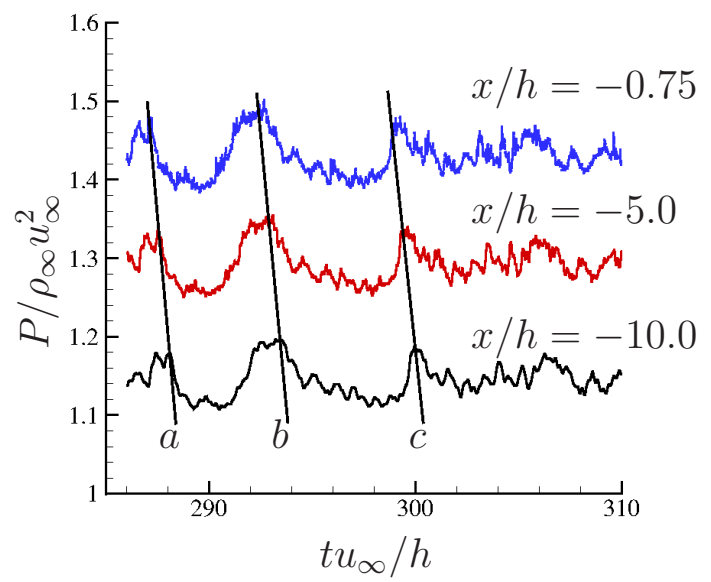

Figure 4: Pressure history upstream of the wedge showing the effect of non-reflecting boundary conditions.

where $p_{v}$ is the vapor pressure. $C_{e}$ and $C_{c}$ are empirical constants whose value is 0.1 [30]. Vapor pressure is related to temperature by

$$
p_{v}=p_{k} \exp \left[\left(1-\frac{T_{k}}{T}\right)\left(a+(b-c T)(T-d)^{2}\right)\right],
$$

where $p_{k}=22.130 \mathrm{MPa}, T_{k}=647.31 \mathrm{~K}, a=7.21, b=1.152 \times 10^{-5}, c=-4.787 \times 10^{-9}$ and $d=483.16$.

To perform LES, Eqs. (1) are first Favre filtered spatially:

$$
\begin{aligned}
\frac{\partial \bar{\rho}}{\partial t} & =-\frac{\partial}{\partial x_{k}}\left(\bar{\rho} \tilde{u}_{k}\right), \\
\frac{\partial \bar{\rho} \tilde{u}_{i}}{\partial t} & =-\frac{\partial}{\partial x_{k}}\left(\bar{\rho} \tilde{u}_{i} \tilde{u}_{k}+\bar{p} \delta_{i k}-\tilde{\sigma}_{i k}-\tau_{i k}\right) \\
\frac{\partial \bar{\rho} \tilde{Y}}{\partial t} & =-\frac{\partial}{\partial x_{k}}\left(\bar{\rho} \tilde{Y} \tilde{u}_{k}-t_{k}\right)+\tilde{S}_{e}-\tilde{S}_{c}, \\
\frac{\partial \bar{\rho} \tilde{e}_{s}}{\partial t} & =-\frac{\partial}{\partial x_{k}}\left(\bar{\rho} \tilde{e_{s}} \tilde{u}_{k}-\tilde{Q}_{k}-q_{k}\right)-\bar{p} \frac{\partial \tilde{u_{k}}}{\partial x_{k}}+\tilde{\sigma_{i k}} \frac{\partial \tilde{u}_{i}}{\partial x_{k}} .
\end{aligned}
$$

Here, the tilde quantities are Favre averaged quantities and $\tau_{i k}, q_{k}$ and $t_{k}$ are subgrid scale (SGS) terms namely: SGS stress, SGS heat flux and SGS scalar flux. These terms are modeled using the Dynamic Smagorinsky model (DSM) :

$$
\begin{aligned}
\tau_{i j}-\frac{\delta_{i j}}{3} \tau_{k k} & =-2 C_{S}(\mathbf{x}, t) \bar{\rho} \Delta^{2}|\tilde{S}| \widetilde{S_{i j}^{*}}, \\
\tau_{k k} & =2 C_{I}(\mathbf{x}, t) \bar{\rho} \Delta^{2}|\tilde{S}|^{2}, \\
q_{i} & =-\bar{\rho} \frac{C_{S}(\mathbf{x}, t) \Delta^{2}|\tilde{S}|}{P r_{T}} \frac{\partial \bar{T}}{\partial x_{i}}, \\
t_{i} & =-\bar{\rho} \frac{C_{S}(\mathbf{x}, t) \Delta^{2}|\tilde{S}|}{S c_{T}} \frac{\partial \bar{Y}}{\partial x_{i}},
\end{aligned}
$$

where $|S|=\sqrt{2 S_{i j} S_{i j}}$ and $S_{i j}^{*}=S_{i j}-1 / 3 S_{k k} \delta_{i j}$. The model coefficients $C_{s}, C_{I}, \operatorname{Pr}_{T}$ and 
$S c_{T}$ are determined using the Germano identity. For example,

$$
\begin{aligned}
C_{S} \Delta^{2} & =\frac{1}{2} \frac{\left\langle L_{i j}^{*} M_{i j}^{*}\right\rangle}{\left\langle M_{i j}^{*} M_{i j}^{*}\right\rangle} \\
L_{i j}^{*} & =\left(\frac{\widehat{\rho u_{i} \cdot \overline{\rho u_{j}}}}{\bar{\rho}}\right)-\frac{\widehat{\rho u_{i}} \cdot \widehat{\rho u_{j}}}{\widehat{\bar{\rho}}} \\
M_{i j}^{*} & =\bar{\rho} \widehat{|\tilde{S}| \widetilde{S_{i j}^{*}}}-\widehat{\bar{\rho}}\left(\frac{\widehat{\Delta}}{\bar{\Delta}}\right)^{2} \widehat{|\widetilde{S}|} \widehat{\widehat{S_{i j}^{*}}}
\end{aligned}
$$

where, $\langle\cdot\rangle$ denotes spatial average over neighboring control volumes and the caret denotes test filtering. Test filtering is defined by the linear interpolation from face values of a control volume, which is again the interpolation from two adjacent cell center values [28]:

$$
\widehat{\phi}=\frac{1}{N_{\text {face }}} \sum_{\text {no of face }} \phi_{f}=\frac{1}{2 N_{\text {face }}} \sum_{\text {no of face }}\left(\phi_{i c v 1}+\phi_{i c v 2}\right),
$$

where $N_{\text {face }}$ is the number of faces for a given control volume.

For unsteady RANS, the Spalart-Allmaras model [36] is used:

$$
\begin{array}{r}
\frac{\partial \rho \tilde{\nu}}{\partial t}+\frac{\partial\left(\rho \tilde{\nu} u_{k}\right)}{\partial x_{k}}=c_{b 1} \tilde{S} \rho \tilde{\nu}+\frac{1}{\sigma}\left[\left(1+c_{b 2}\right) \nabla \cdot((\rho \nu+\rho \tilde{\nu}) \nabla \tilde{\nu})-c_{b 2}(\rho \nu+\rho \tilde{\nu}) \nabla \cdot \nabla \tilde{\nu}\right] \\
-\rho c_{w 1} f_{w}\left(\frac{\tilde{\nu}}{d}\right)^{2}
\end{array}
$$

where $\nu_{T}=\tilde{\nu} f_{v 1}, f_{v 1}=\chi^{3} /\left(\chi^{3}+c_{v 1}\right)$ and $\chi=\tilde{\nu} / \nu . S$ is the strain rate. The model is closed with the following coefficients and wall functions:

$$
\begin{gathered}
\tilde{S}=S+\frac{\tilde{\nu}}{\kappa^{2} d^{2}} f_{v 2}, f_{v 2}=\left(1+\frac{\chi}{c_{v 2}}\right)^{-3}, \\
f_{w}=g\left(\frac{1+c_{w 3}^{6}}{g^{6}+c_{w 3}^{6}}\right)^{1 / 6}, g=r+c_{w 2}\left(r^{6}-r\right), r=\frac{\tilde{\nu}}{\tilde{S} \kappa^{2} d^{2}}, \\
c_{b 1}=0.1355, \sigma=\frac{2}{3}, c_{b 2}=0.622, \kappa=0.41, c_{v 2}=5, \\
c_{w 1}=\frac{c_{b 1}}{\kappa^{2}}+\frac{1+c_{b 2}}{\sigma}, c_{w 2}=0.3, c_{w 3}=2, c_{v 1}=7.1 .
\end{gathered}
$$

For cavitating flows, Coutier-Delgosha et al. [7] observed that the eddy viscosity obtained from standard RANS models can be excessive, especially near the cavity closure region, which prevents cloud formation. Hence they suggested to modify the eddy viscosity near the cavity interface as

$$
\mu_{T}=\nu_{T}\left[\rho_{g}+\left(\rho_{l}-\rho_{g}\right)(1-\alpha)^{10}\right] .
$$

Once $\nu_{T}$ is computed, the Reynolds stress is given by

$$
\mathcal{R}_{i j}=-2 \rho \nu_{T} \bar{S}_{i j}
$$

The turbulent thermal conductivity and turbulent scalar diffusivity are also computed from the eddy viscosity assuming a turbulent Prandtl number $\left(P r_{t}\right)$ of 0.9 and a turbulent Schmidt number $\left(S c_{t}\right)$ of 0.7 . The turbulent scalar equation is then modified as

$$
\frac{\partial \rho Y}{\partial t}=-\frac{\partial}{\partial x_{k}}\left(\rho Y u_{k}\right)+S_{e}-S_{c}+\frac{\partial}{\partial x_{k}}\left(\frac{\nu_{t}}{S c_{t}} \frac{\partial \rho Y}{\partial x_{k}}\right)
$$




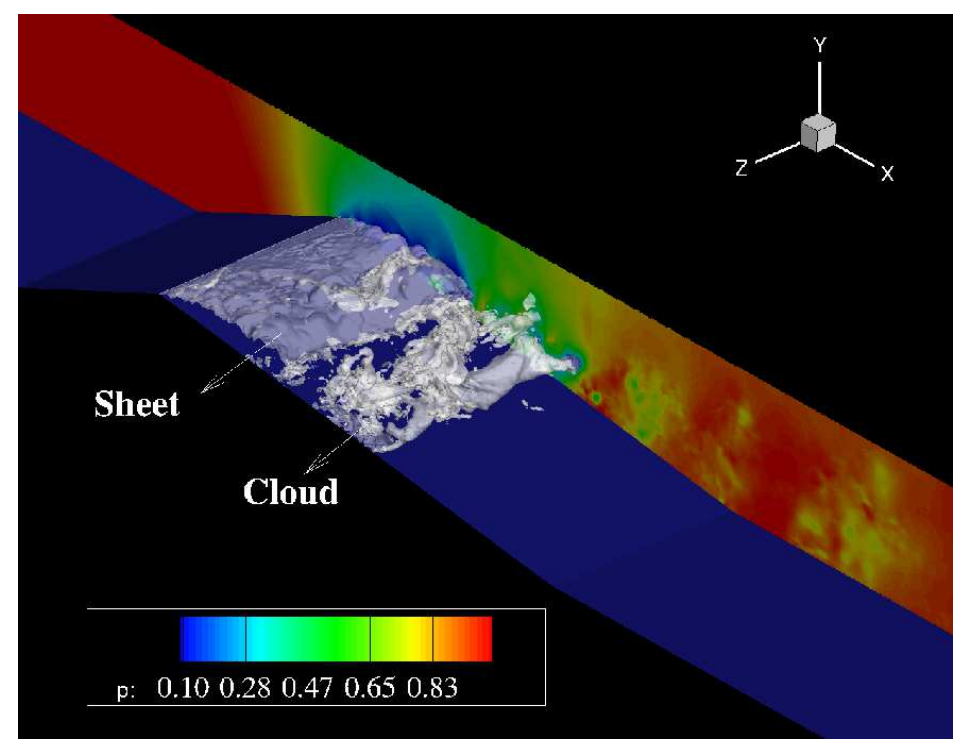

$(a)$

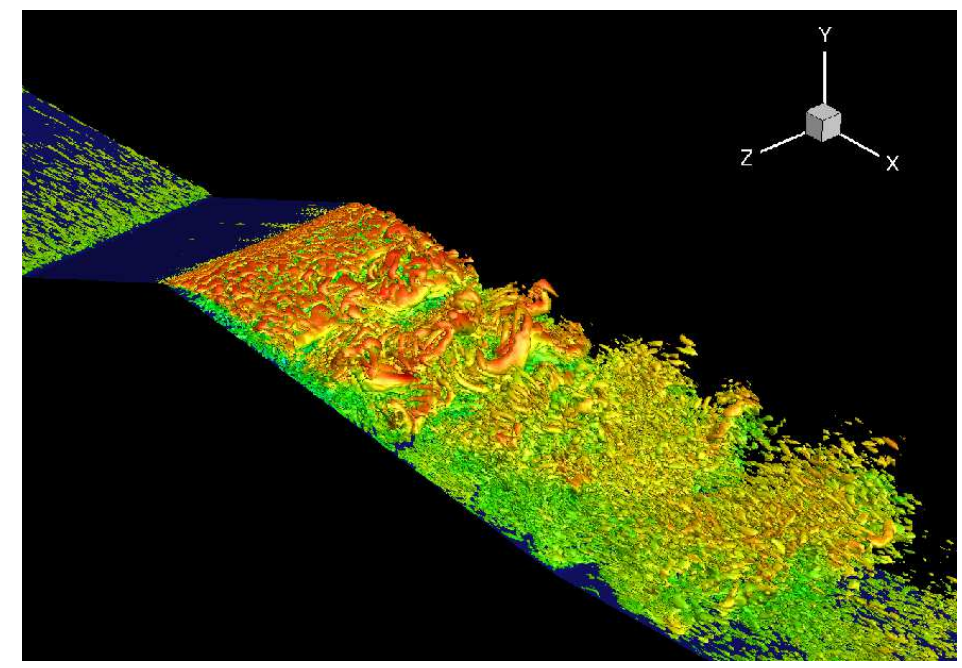

(b)

Figure 5: (a) Instantaneous void fraction contours showing sheet and cloud cavities, (b) Isocontours of Q-criterion showing vortical structures. 
The simulations use the algorithm developed by Gnanaskandan and Mahesh [15] to simulate cavitating flows on unstructured grids. The algorithm makes use of a novel predictor corrector approach. In the predictor step, the governing equations are discretized using a symmetric non dissipative scheme, where the fluxes at a cell face are given by

$$
\phi_{f_{c}}=\frac{\phi_{i c v 1}+\phi_{i c v 2}}{2}+\frac{1}{2}\left(\left.\nabla \phi\right|_{i c v 1} \cdot \Delta \mathbf{x}^{i c v 1}+\left.\nabla \phi\right|_{i c v 2} \cdot \Delta \mathbf{x}^{i c v 2}\right)
$$

where $\Delta \mathbf{x}^{i c v 1}=\mathbf{x}_{f_{c}}-\mathbf{x}_{i c v 1}$, and $\left.\nabla \phi\right|_{i c v 1}$ denotes the gradient defined at $i c v 1$, which is computed using a least squares method. The viscous fluxes are split into compressible and incompressible contributions and treated separately. Once the fluxes are obtained, a predicted value $\widehat{q}_{j}^{n+1}$ is computed using an explicit Adams-Bashforth scheme.

$$
\widehat{q}_{j}^{n+1}=\widehat{q}_{j}^{n}+\frac{\Delta t}{2}\left[3 \operatorname{rhs}_{j}\left(\widehat{\mathbf{q}}^{n}\right)-\operatorname{rhs}_{j}\left(\widehat{\mathbf{q}}^{n-1}\right)\right],
$$

where $\mathrm{rhs}_{j}$ denotes the $j^{\text {th }}$ component of the right hand side of the governing equations, and the superscript $n$ denotes the $n^{t h}$ time step. The final solution $q_{j}^{n+1}$ at $t+\Delta t$ is obtained from a corrector scheme

$$
q_{j, c v}^{n+1}=\widehat{q}_{j, c v}^{n+1}-\frac{\Delta t}{V_{c v}} \sum_{\text {faces }}\left(F_{f}^{*} \cdot n_{f}\right) A_{f},
$$

where $F_{f}^{*}$ is the filter numerical flux of the following form:

$$
F_{f c}^{*}=\frac{1}{2} R_{f c} \Phi_{f c}^{*}
$$

Here $R_{f c}$ is the right eigenvector vector at the face computed using Roe-average of the variables from left and right control volumes. The expression for the $l$ th component of $\Phi^{*}, \phi^{* l}$ is given by

$$
\phi_{f c}^{* l}=k \theta_{f c}^{l} \phi_{f c}^{l},
$$

where $k$ is an adjustable parameter and $\theta_{f c}$ is the Harten's switch function given by

$$
\theta_{f c}=\sqrt{0.5\left(\widehat{\theta}_{i c v 1}^{2}+\widehat{\theta}_{i c v 2}^{2}\right)}, \widehat{\theta}_{i c v 1}=\frac{\left|\beta_{f c}\right|-\left|\beta_{f 1}\right|}{\left|\beta_{f c}\right|+\left|\beta_{f 1}\right|}, \widehat{\theta}_{i c v 2}=\frac{\left|\beta_{f 2}\right|-\left|\beta_{f c}\right|}{\left|\beta_{f 2}\right|+\left|\beta_{f c}\right|} .
$$

Here, $\beta_{f}=R_{f}^{-1}\left(q_{i c v 2}-q_{i c v 1}\right)$ is the difference between characteristic variables across the face. For $\phi^{\ell}$, the Harten-Yee TVD form is used as suggested by [39].

$$
\begin{aligned}
\phi_{f_{c}}^{\ell} & =\frac{1}{2} \Psi\left(a_{f_{c}}^{\ell}\right)\left(g_{i c v 1}^{\ell}+g_{i c v 2}^{\ell}\right)-\Psi\left(a_{f_{c}}^{\ell}+\gamma_{f_{c}}^{\ell}\right) \beta_{f_{c}}^{\ell}, \\
\gamma_{f_{c}}^{\ell} & =\frac{1}{2} \frac{\Psi\left(a_{f_{c}}^{\ell}\right)\left(g_{i c v 2}^{\ell}-g_{i c v 1}^{\ell}\right) \beta_{f_{c}}^{\ell}}{\left(\beta_{f_{c}}^{\ell}\right)^{2}+\epsilon}
\end{aligned}
$$

where $\epsilon=10^{-7}$ and $\Psi(z)=\sqrt{\delta+z^{2}}(\delta=1 / 16)$ is introduced for entropy fixing. $a_{f_{c}}^{\ell}$ is an element of the Jacobian matrix. [28] and [15] proposed a modification to the Harten's switch to accurately represent under-resolved turbulence for single phase and multi phase flow mixtures respectively by multiplying $\theta_{f_{c}}$ with $\theta_{f_{c}}^{\star}$ given by

$$
\begin{aligned}
\theta_{f_{c}}^{\star} & =\frac{1}{2}\left(\theta_{i c v 1}^{\star}+\theta_{i c v 2}^{\star}\right)+\left|\left(\alpha_{\mathrm{icv} 2}-\alpha_{\mathrm{icv} 1}\right)\right|, \\
\theta_{i c v 1}^{\star} & =\frac{(\nabla \cdot \mathbf{u})_{i c v 1}^{2}}{(\nabla \cdot \mathbf{u})_{i c v 1}^{2}+\Omega_{i c v 1}^{2}+\epsilon} .
\end{aligned}
$$




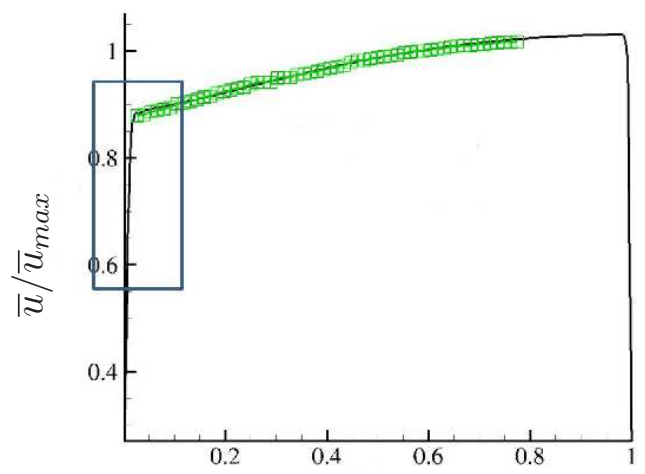

(a) $y / h$

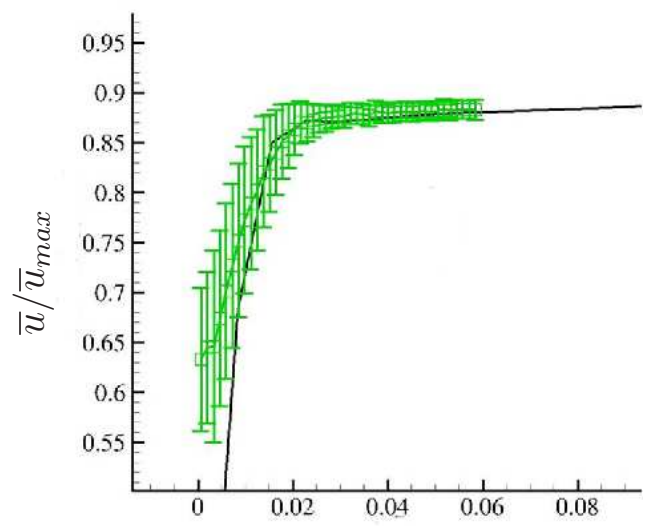

(b)

$$
y / h
$$

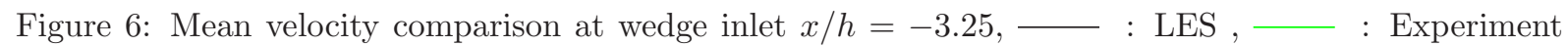
(Ganesh [13]). (b) shows the close-up view of near wall region as illustrated in $(a)$.

Gnanaskandan and Mahesh [15] have evaluated this algorithm for a variety of flows including a cavitating shock tube, turbulent cavitating flow over a hydrofoil and a hemispherical headform [14].

\section{Problem Description}

Figure 1 shows a schematic of the computational domain. The mean flow is along the $x$-axis and the wedge apex is located at the origin. The height of the wedge is $h=1$ inch. The computational domain is extended in both upstream (25h) and downstream directions (50h) to minimize the effect of acoustic reflection from the boundaries. Velocity and pressure are prescribed at the inlet and downstream pressure is prescribed at the outlet. The boundary conditions are iteratively changed in order to match the average cavitation number obtained from the experiment at the $y-z$ plane located at $x / h=-3.25$ just before the converging section starts. No slip boundary conditions are imposed on top and bottom walls. Periodic boundary conditions are enforced at the spanwise boundaries. Acoustically absorbing boundary conditions [6] are applied in sponge layers at both inlet and outlet as shown in Figure 1 . The term $-\gamma\left(\vec{q}-\vec{q}_{r e f}\right)$ is added to the governing equations, where $\gamma$ is zero outside the sponge layer, $\vec{q}$ denotes the vector of conservative variables and the subscript 'ref' denotes the reference solution to which the flow inside the sponge layer is damped. Velocity and pressure at the inlet sponge are damped to the inlet values, while only the thermodynamic variables are damped to the downstream value at the outlet. The Reynolds number of the flow based on the wedge height ( $h=1 \mathrm{inch}$ ) and a bulk velocity of $7.9 \mathrm{~m} / \mathrm{s}$ is approximately $0.2 \times 10^{6}$. The computational mesh at the inlet, outlet and test sections are shown in Figure 2. The mesh is made very fine near the wedge apex and along the entire length of the wedge where the major portion of the vapor is expected to form. The minimum grid spacing near the wedge is $0.001 \mathrm{~h} \times 0.001 \mathrm{~h} \times 0.01 \mathrm{~h}$ in the wall normal, streamwise and spanwise directions respectively. The wall normal spacing stretches to $0.005 h$ at a height of $0.5 h$ from the wedge apex and further to about $0.01 h$ at a height of $h$ from the apex. In the streamwise direction, the grid is stretched to $0.02 h$ at a distance $3.5 h$ from the apex and further to $0.01 h$ at the end of the wedge. The total number of control volumes is approximately 45 million. The non-dimensional time step used in the simulation is $t u_{\infty} / h=1 \times 10^{-5}$. The simulation is initialized using a two dimensional solution obtained from a RANS simulation performed using Spalart-Allmaras 


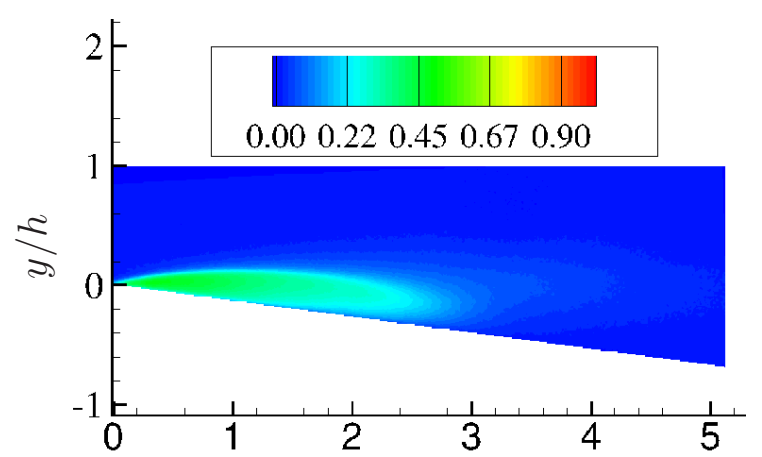

(a)

$$
x / h
$$

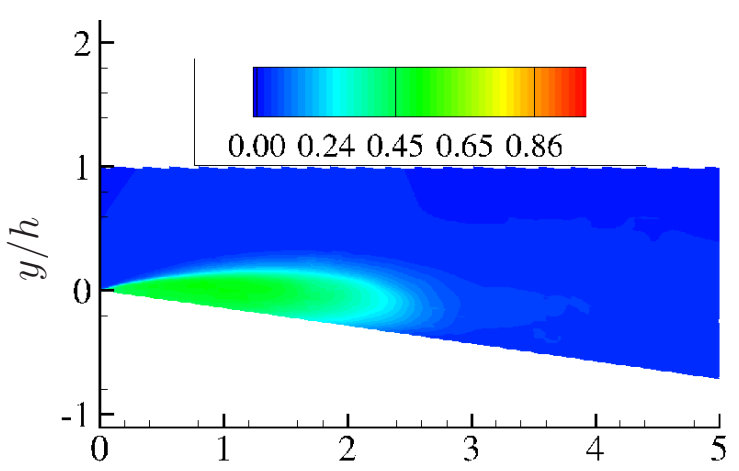

(b)

Figure 7: Comparison of mean void fraction contours, (a) Experiment (Ganesh [13]) (b) LES.
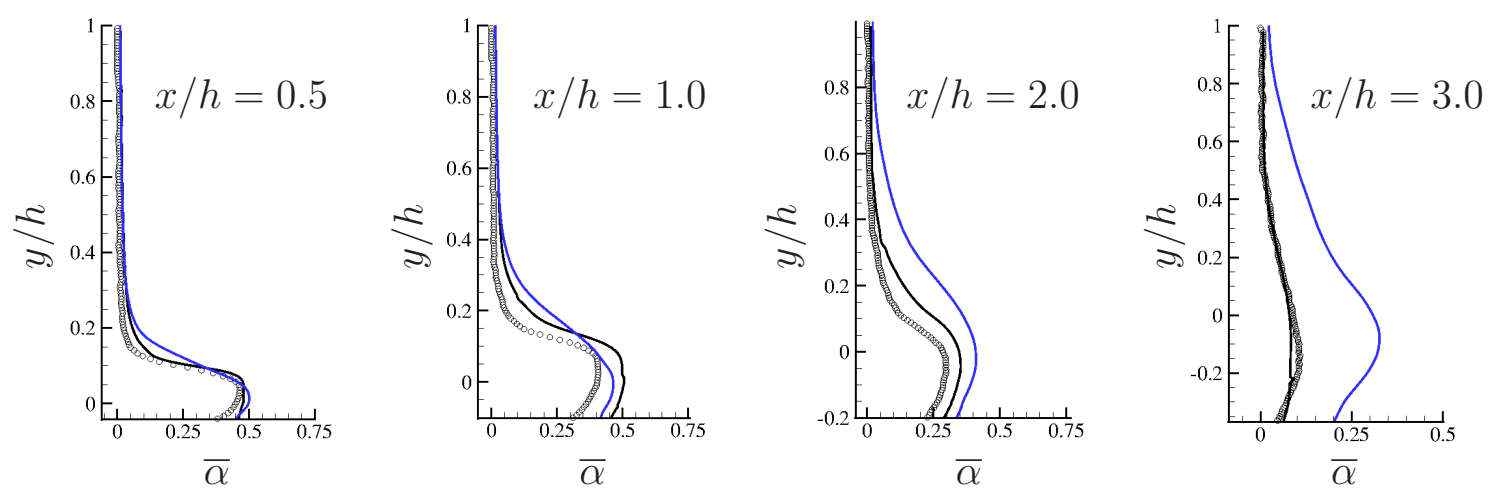

Figure 8: Comparison of mean void fraction profiles at different streamwise locations, $\circ:$ Experiment (Ganesh [13]), — : LES, — : unsteady RANS.

model [36] under the same conditions prescribed for the LES simulation. The cavitation number obtained in the experiments at $x / h=-3.25$ is 2.0 and that in the simulation is 2.1. The cavitation number obtained in simulation is using a pressure of $69 \mathrm{KPa}$ and a velocity of $7.9 \mathrm{~m} / \mathrm{s}$ while the corresponding values in the experiments are $66 \mathrm{KPa}$ and $8 \mathrm{~m} / \mathrm{s}$. Localization of dissipation is essential to accurately simulate turbulent flows. To assess this effect, the filter flux $\frac{F_{f c}^{*}}{V_{c v}}$ is computed and plotted in Figure 3 for the continuity equation, where $F_{f c}^{*}=\frac{1}{2} R_{f c} \Phi_{f c}^{*}$ as defined in Section 2. From Figure 3, it can be observed that the dissipation is significant only at the cavity interface, cavity closure and inside the cloud cavity. The dissipative fluxes for other equations also show similar behavior and hence are not shown here. In order to illustrate that pressure waves traveling towards inlet are not reflected back into the domain, we plot time history of pressure at three different locations upstream of the wedge in Figure 4. The major pressure rise events tagged as $a, b$ and $c$ indicate that the direction of pressure waves is towards the inlet and that no waves are reflected back into the domain.

\section{Results}

The nature of the instantaneous solution is illustrated using isocontours of void fraction in Figure 5(a) which shows the presence of both sheet and cloud cavities. Pressure drops in the converging portion as the flow accelerates and cavity develops at the wedge apex as the instantaneous pressure drops below vapor pressure. This cavity initially remains attached to the wedge and grows along the flow direction and is termed as sheet cavity. The 

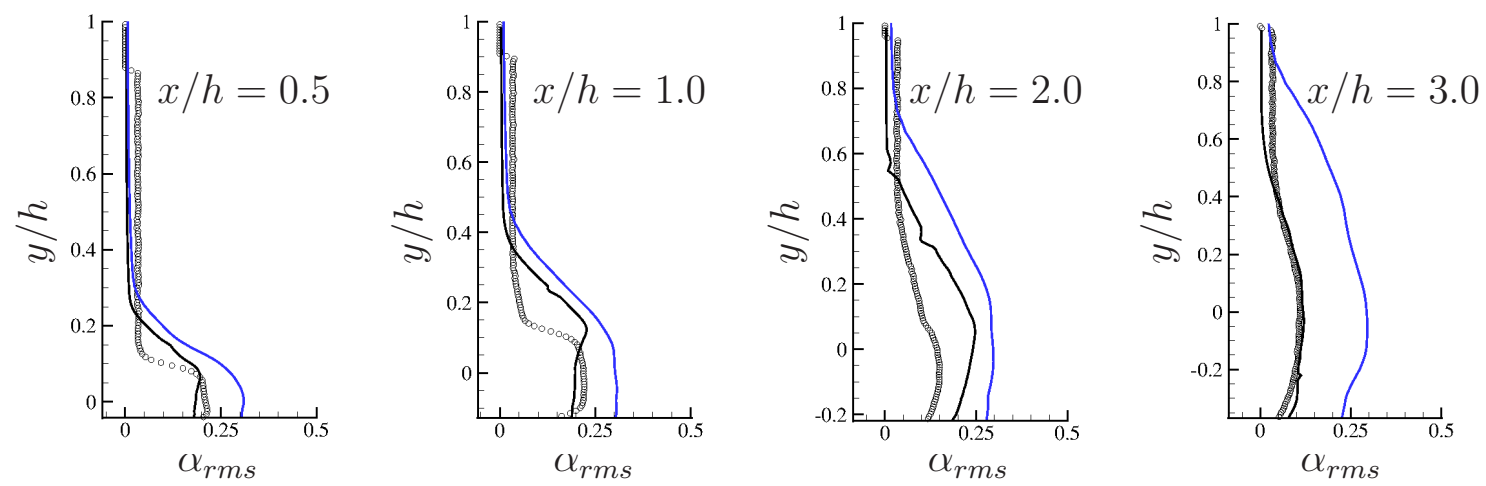

Figure 9: Comparison of RMS of void fraction profiles at different streamwise locations, o : Experiment (Ganesh [13]), — : LES, — : unsteady RANS.

sheet cavity on reaching a critical length, breaks into a cloud. This cloud is highly three dimensional and it is the collapse of this cloud that causes noise, vibration and surface erosion. The vertical plane in Figure 5(a) shows pressure contours showing low pressure near the cavity region and high pressure downstream. Small patches of low pressure downstream are the convected cloud cavities. Pressure exhibits both cyclic behavior (close to leading edge of the cavity) and a highly transient (small scale) behavior (downstream of the cloud). The turbulent nature of the flow downstream prevents a coherent wavelike behavior there, since turbulence breaks up the wave thus causing highly intermittent pressure fluctuations. The pressure waves produced on cavity collapse impinge on the developing sheet cavity and the smaller secondary cavities. Figure 5(b) shows isocontours of Q-criterion colored with streamwise velocity. The intensely vortical nature of the flow and wide range of length scales are apparent in both sheet and cloud regions. The presence of sheet and cloud is also evident from the vortical structures in the flow, where the sheet cavity has larger structures due to its relatively two-dimensional nature while the cloud has both large and small structures. Note that the vorticity in the cloud persists even after the cloud collapses.

\subsection{Comparison to experiment}

We compare the mean inlet velocity profile, mean and RMS void fraction and the Strouhal number corresponding to cavity auto-oscillation. The averaged statistics presented in this section are obtained by performing a time average over $40 \mathrm{th} / \mathrm{u}_{\infty}$ (about four shedding cycles) and has approximately 30,000 samples. The separation between the samples is $0.001 t h / u_{\infty}$ which captures all relevant high frequencies. The time averaged results are then averaged along the spanwise direction to further improve convergence of the statistics. The mean inlet velocity profile obtained from the simulation is first compared to the experiment to ensure that the oncoming velocity profile to the wedge is predicted properly. Figure 6(a) shows the overall comparison obtained between simulation and experiments and Figure 6(b) shows a close-up view of the velocity profile near the bottom wall. Overall good agreement is obtained. Figure 7 compares the mean void fraction contours obtained from experiment and LES. Note the overall good agreement of the cavity length and the value of mean void fraction inside the cavity. The cavity thickness predicted by the simulation is slightly larger than the experimental measurement. The mean void fraction at different streamwise locations on the wedge obtained from LES are compared to the experimental results in Figure 8. Also shown are results obtained using unsteady RANS simulation. The unsteady RANS simulations are performed using Spalart-Allmaras model [15] with modified eddy viscosity [8]. Note the overall good 

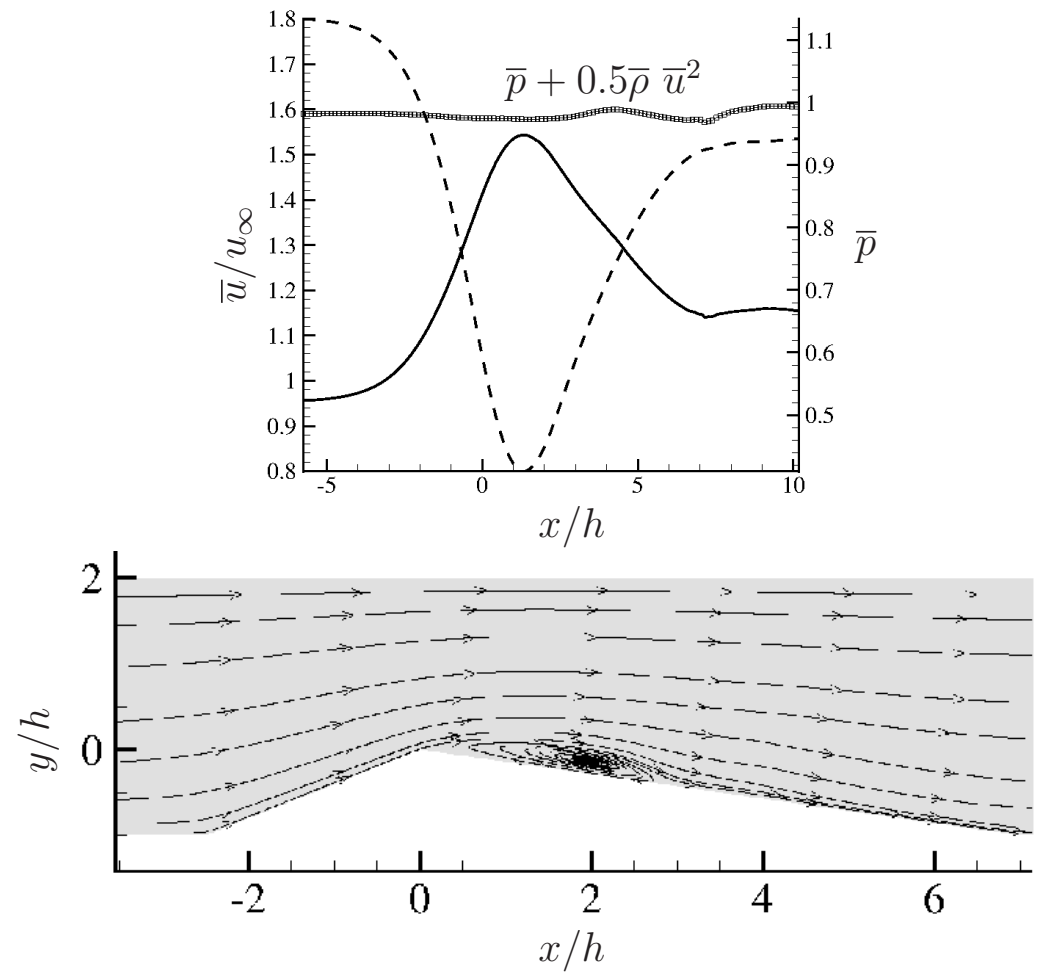

Figure 10: (a)Variation of mean velocity and pressure along streamwise direction, $\_$: $u$-velocity and --- : pressure, (b) Mean streamlines.

agreement of the value of mean void fraction inside the cavity for LES in contrast to unsteady RANS. Further, the length of the cavity is also not predicted well by unsteady RANS, while LES gives an excellent agreement for the mean length. The thickness is slightly mispredicted by LES at stations $x / h=1.0$ and $x / h=2.0$, while the thickness predicted by unsteady RANS is even worse. Overall, LES agrees much better with the experiments than unsteady RANS. No error bars are available from experiment for mean void fraction data. Next we compare the RMS of void fraction obtained from simulations and experiment in Figure 9. Note that error bars are not available from the experiment for this quantity either, and that only the resolved portion of the fluctuation obtained from LES is shown here. The free stream fluctuation measured in the experiment does not go to zero while that predicted by LES goes to zero away from the cavity. The qualitative trend from LES agrees well with the experiment at all the stations and LES also seems to predict the RMS much better than unsteady RANS. The fact that LES predicts a thicker cavity is also manifested in the form of higher magnitude of fluctuations away from the wedge. Overall, the comparisons for void fraction data are encouraging suggesting the suitability of LES in predicting this highly unsteady phenomenon. The Strouhal number corresponding to shedding frequency obtained from the numerical simulation is $S t=0.28$, which is computed from the time histories of pressure and void fraction at several locations inside and downstream of the mean cavity and will be discussed in Section 4.3. This value lies within the range of $0.25-0.3$ obtained in the experiment.

\subsection{Mean pressure, density and velocity field}

Figure 10(a) shows the variation of mean velocity and pressure along a streamline at $y / h=1$. The flow accelerates in the converging portion (upto $x / h=0$ ) and pressure drops correspondingly. The presence of the cavity accelerates the free stream flow further due 


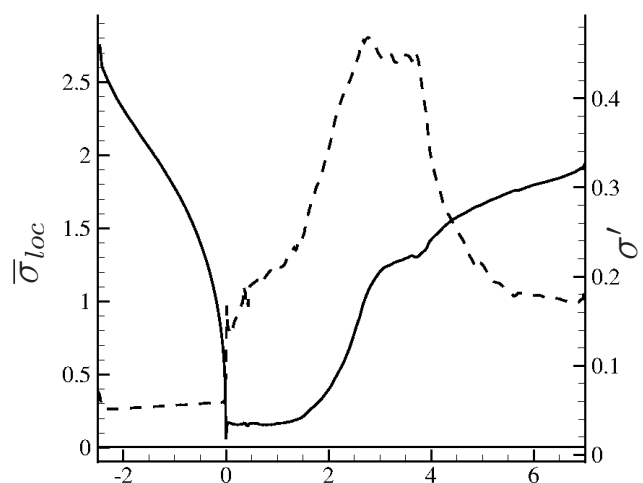

(a)

$x / h$

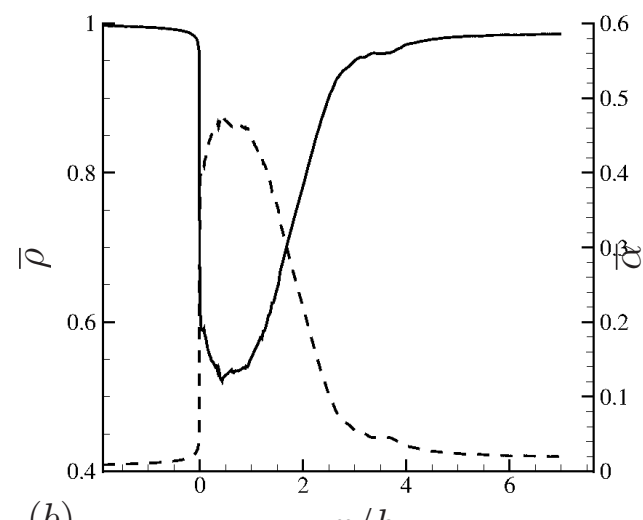

(b)

$x / h$

Figure 11: (a) Variation of $\bar{\sigma}_{l o c}(-)$ and $\sigma^{\prime}(----)$ along the wedge wall, $(b)$ Variation of mean density ( - ) and mean void fraction (---- ) along the wedge wall.

to the confining effect of the wall, upto about $x / h=1.5$ after which the flow decelerates. Also shown in the figure is $\bar{p}+0.5 \bar{\rho} \bar{u}^{2}$, which remains nearly constant showing that the viscous effects are confined close to the wall. The mean streamlines (Figure 10(b)) show a reverse flow region which corresponds to the cavity location. The separation streamline that separates the reverse flow inside the cavity from the flow outside the cavity stagnates at the cavity closure. The adverse pressure gradient at this closure location causes a reverse flow to penetrate the cavity, which contributes to cloud formation.

We define, $\bar{\sigma}_{l o c}=2\left(\bar{p}-p_{v}\right) / \rho_{\infty} u_{\infty}^{2}$ and $\sigma^{\prime}=2 \sqrt{\overline{p^{\prime 2}}} / \rho_{\infty} u_{\infty}^{2}$ to further quantify the mean and RMS characteristics of the vapor. Figure 11(a) shows the variation of $\bar{\sigma}_{l o c}$ and $\sigma^{\prime}$ along the wedge. $x / h=0$ is the apex region and minimum $\bar{\sigma}_{l o c}$ is obtained there. It is interesting to see that the mean pressure never falls below the vapor pressure, but the fluctuations at the apex are large enough for the instantaneous local pressure to fall below vapor pressure. Note that the value of RMS of pressure is maximum $x / h=2.5$ which corresponds to the mean closure location of the cavity. This behavior points to cavity oscillation about that position. Figure 11(b) shows the variation of mean density and mean volume fraction along the wedge. It is clear that inception occurs at the apex and the maximum amount of vapor in the mean flow occurs inside the sheet cavity. The region corresponding to the cloud has lesser void fraction than that in the sheet. This observation is also in line with the observations of Coutier-Delgosha et al. [8]

The boundary layer evolution is illustrated using tangential velocity profiles at several streamwise locations as shown in Figure 12(a). Figure 12(b) shows the mean boundary layer profiles in the converging portion. Here, $\overline{u_{t}}$ is the mean velocity tangential to the wall and $d$ is the distance normal to the wall. The acceleration of free stream and thinning of the boundary layer is evident. The velocity at the station $x / h=-0.5$ reduces as we move away from the bottom wall. Since this station has the least cross sectional area among the all the stations shown, the effect of top wall is predominant which can be seen in the form of deceleration of the bulk flow away from the wall. Figure 12(c) shows the mean boundary layer profiles in the diverging portion. The first three stations are inside the mean cavity and the last station is just outside the mean cavity. Negative tangential velocity is seen inside the cavity at the first three stations. Also shown are mean void fraction profiles to illustrate the relative thickness of the reverse flow inside the cavity with respect to the cavity thickness. A re-entering jet may be defined as a region of relatively high density fluid moving towards the leading edge of the cavity. The thickness 


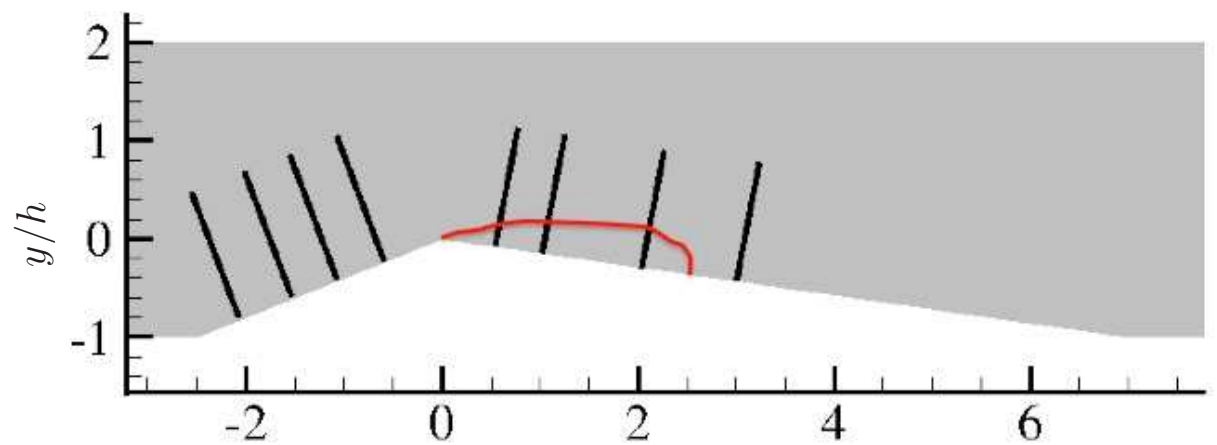

(a)

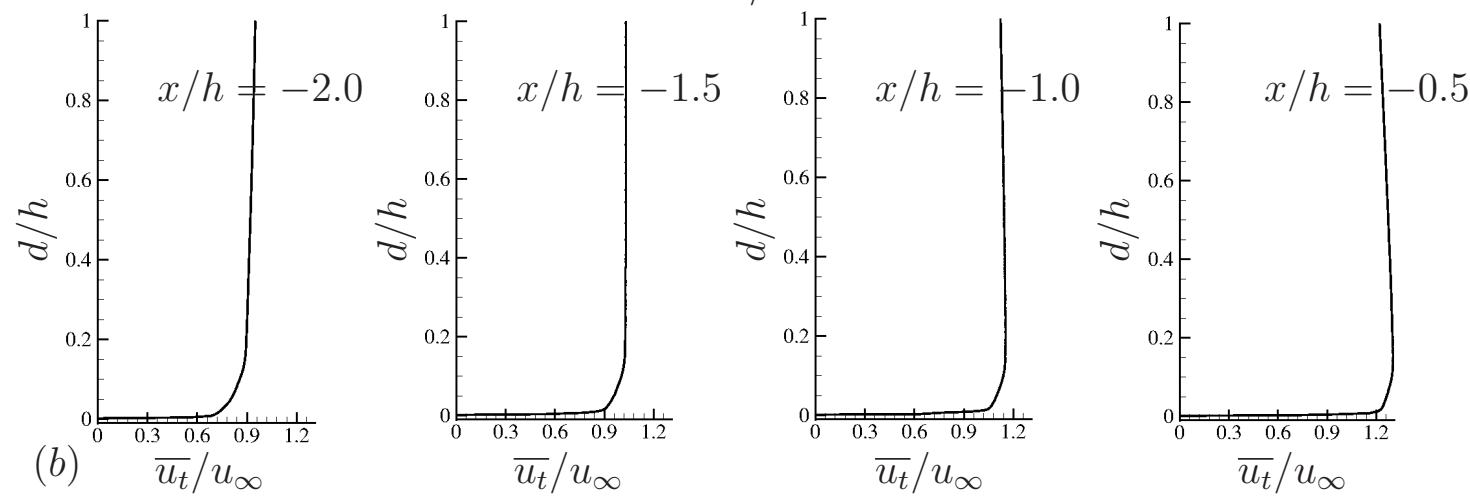

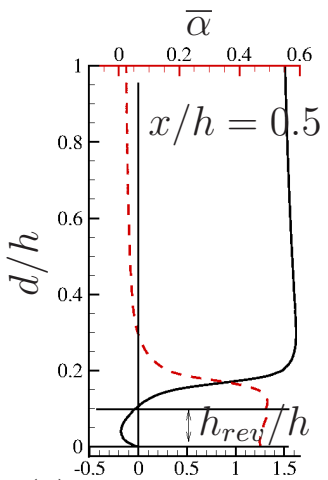

(c)
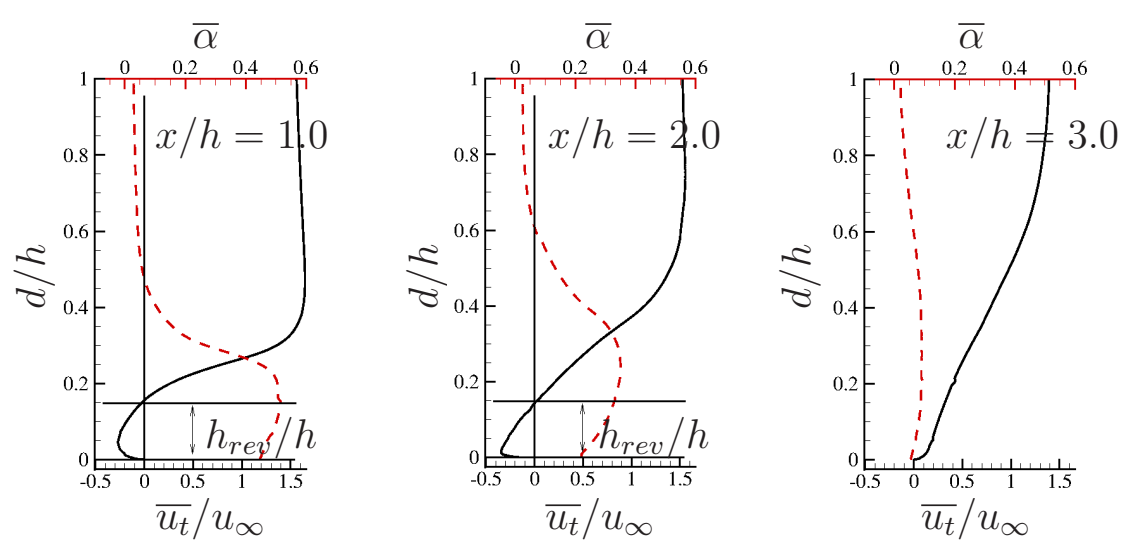

Figure 12: Mean boundary layer profiles at different streamwise locations. - - - : Mean void fraction $(\bar{\alpha})$ and - : Mean tangential velocity $\left(\overline{u_{t}}\right)$. 

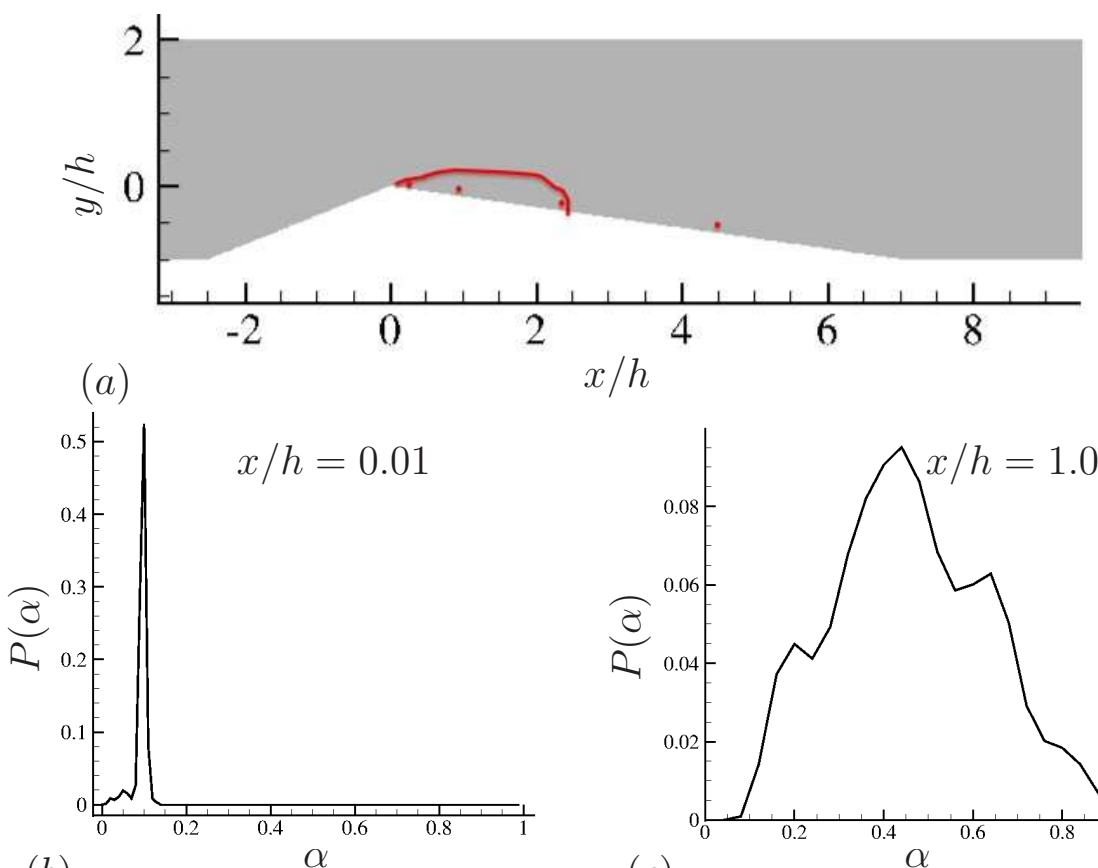

$x / h$

(b)

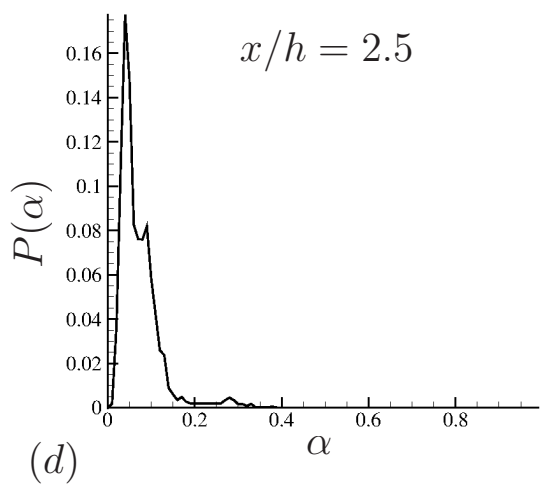

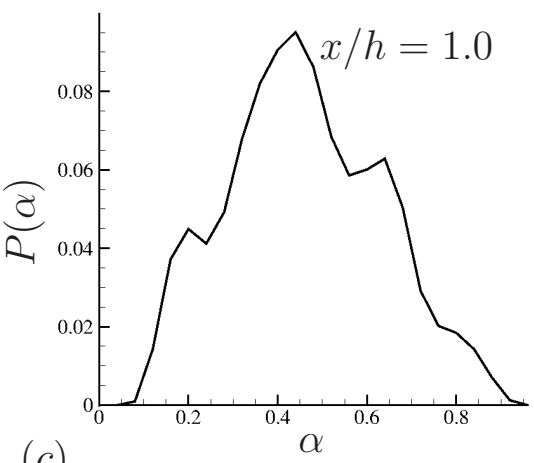

(c)

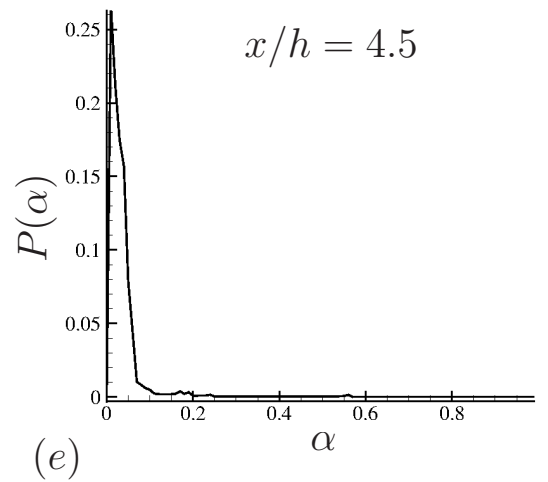

Figure 13: PDF of void fraction at various points inside and outside mean cavity.

of this re-entering jet $h_{\text {rev }} / h$ is also shown in Figure $12(\mathrm{c})$ and is computed to be 0.1 at the station $x / h=0.5$ and 0.15 at the stations $x / h=1.0$ and 2.0. The adverse pressure gradient causes the magnitude of the reverse flow to be maximum at $x / h=2.0$ which further reduces in magnitude as it moves towards the leading edge of the cavity. The magnitude of this negative velocity plays an important role in determining the location where the sheet cavity pinches off to a cloud.

The magnitude of void fraction inside the cavity as observed in Figure 12(c) has a maximum value of about 0.5. A more detailed understanding of the evolution of void fraction is obtained by computing its probability density function. Figure 13 shows the PDF of void fraction at several locations inside and outside the cavity. Figure 13(a) illustrates the location of points chosen for computing the PDFs. The mean cavity profile is illustrated using the red curve. The PDF close to leading edge of the cavity (at $x / h=$ 0.001 ) shows a high probability for $\alpha=0.1$. Since this is the cavity inception region, the value of void fraction here is not expected to be high. It is interesting to note that the time averaged value of void fraction at this location is 0.01 . Thus the most probable value of void fraction at that location is very different from the mean value, illustrating the highly unsteady nature of the flow. As we move downstream along the cavity, the 

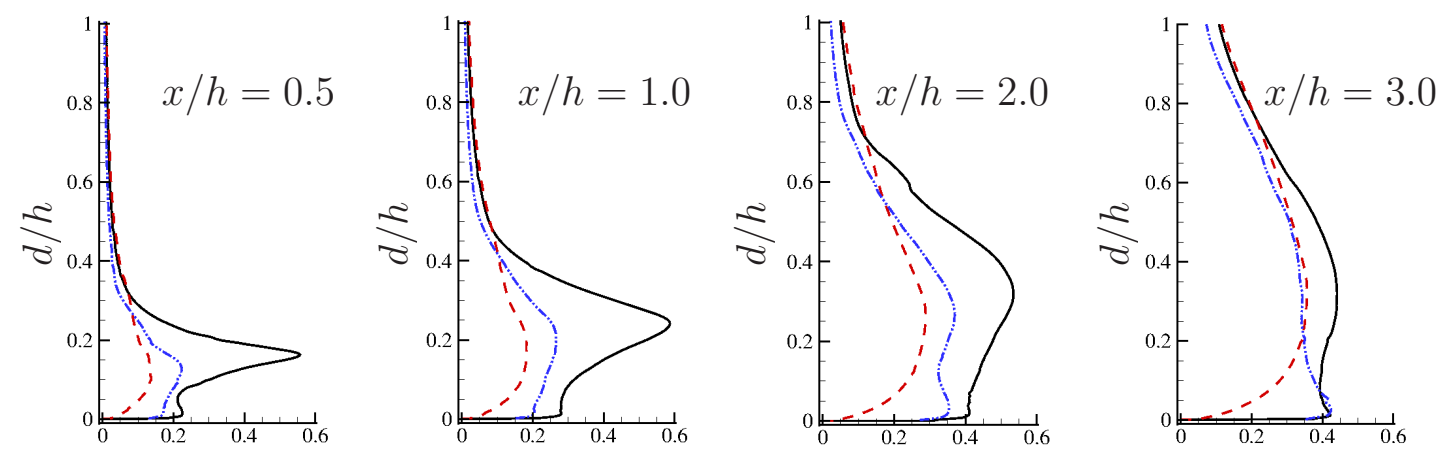

Figure 14: RMS of velocity profiles at different streamwise location, $\sqrt{u_{n}^{\prime} u_{n}^{\prime}} / u_{\infty},-\cdot \cdot-\sqrt{w^{\prime} w^{\prime}} / u_{\infty}$

PDF at $x / h=1.0$ shows a wider distribution with finite probability of occurrence for a large range of void fraction. Larger values of void fraction are more probable during the sheet cavity growth phase and smaller values during the cloud shedding phase. The mean void fraction value at this location is 0.46 , which is also close to the most probable value. The location $x / h=2.5$ corresponds to the mean cavity closure and this location is highly affected by the cavity unsteadiness. The two most probable values of void fraction here are 0.05 and 0.1 . Note that these values differ significantly from the time averaged value of 0.01 there and the occurrence of 0.1 corresponds to the passage of a cloud. Finally the location at $x / h=4.5$ in the wake of the cavity shows that the most probable value of void fraction there is close to the free-stream void fraction $\alpha_{0}=0.01$. However, the passage of clouds at periodic intervals means that there is also a finite probability of void fraction around 0.1. The void fraction content in a cloud is low as observed by Coutier-Delgosha et al. [8] and it drops further as the cloud convects to a high pressure region.

Figure 14 shows the turbulent velocity profiles at the stations discussed in Figure 12. Note that we show only the resolved fluctuations. Within the cavity at $x / h=0.5$ and 1.0 , the fluctuations along the tangential direction dominate the other two components. However, near the cavity closure and downstream of the cavity, all three components of fluctuations are equally significant. This indicates that the flow near the cavity closure and in the cavity wake are highly three-dimensional compared to that inside the cavity.

\subsection{Time evolution of cavity growth and collapse}

The time evolution of sheet to cloud transition is shown as a series of snapshots in Figure 15. On the left are instantaneous span-averaged contours of void fraction and on the right are the instantaneous void fraction contours from the symmetry plane. The entire cycle can be roughly divided into five different events and each figure in Figure 15 is representative of a particular event. The events are : (a) growth of sheet cavity to its full length, (b) primary cloud pinch off, (c) primary cloud shedding and cavity regrowth, (d) and (e) secondary cloud shedding and (f) sheet cavity regrowth. The cavity first grows to an average length of $x / D=2.0$. The instantaneous symmetry plane contours show the impending pinch off at the trailing edge of sheet cavity. The cavity then pinches off and the primary cloud sheds as shown in the span-averaged contours. The symmetry plane contours do not show a clear pinch off indicating that the cloud is still attached to the sheet in the symmetry plane. The stark contrast between the span-averaged and instantaneous contours indicates the three-dimensionality of the cavity. The cavity then regrows to about $50 \%$ of the full cavity length and a secondary cloud pinching occurs. This phenomenon is highly three-dimensional and the instantaneous contours show the 

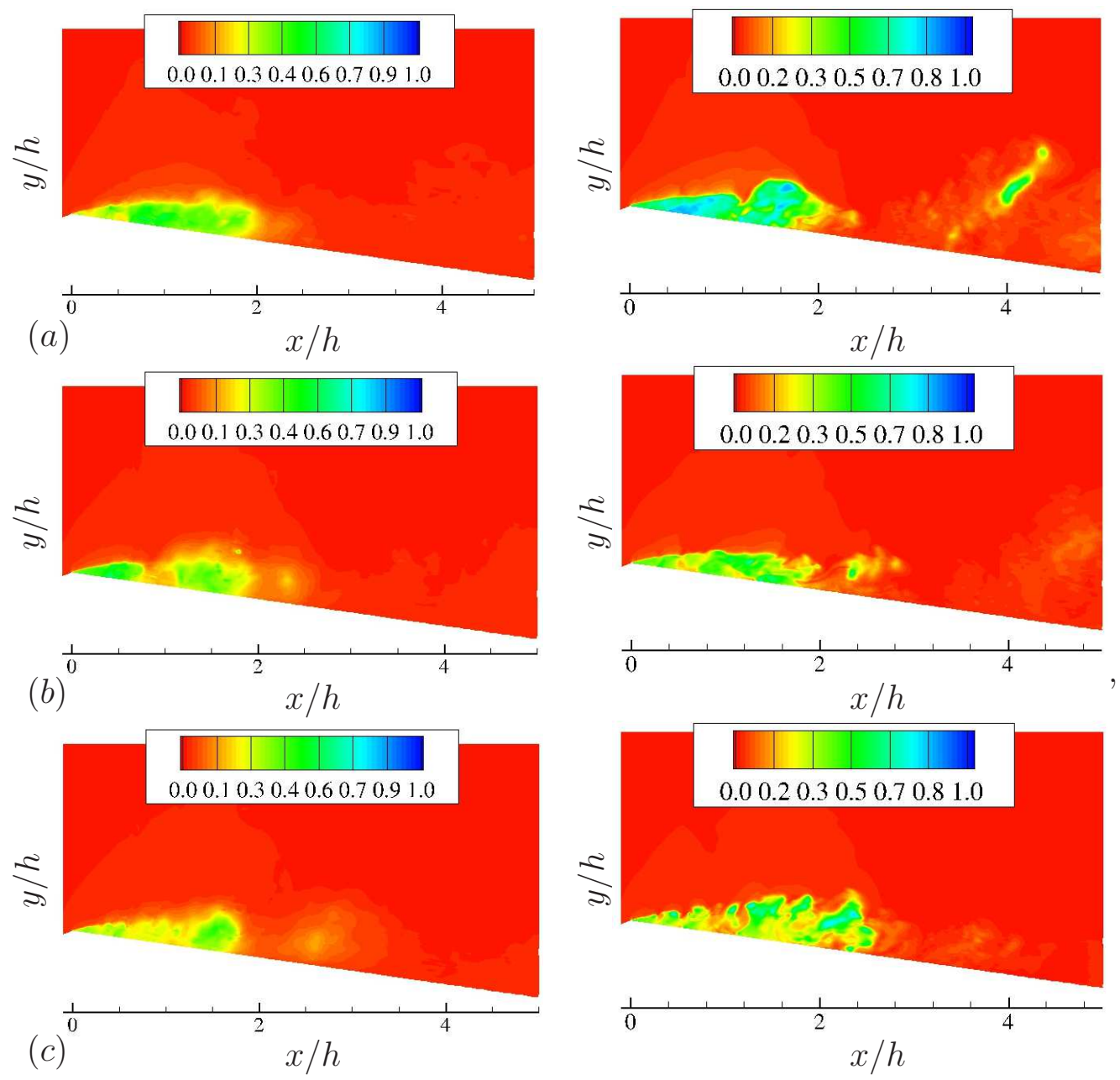

Figure 15: See next page for caption. 

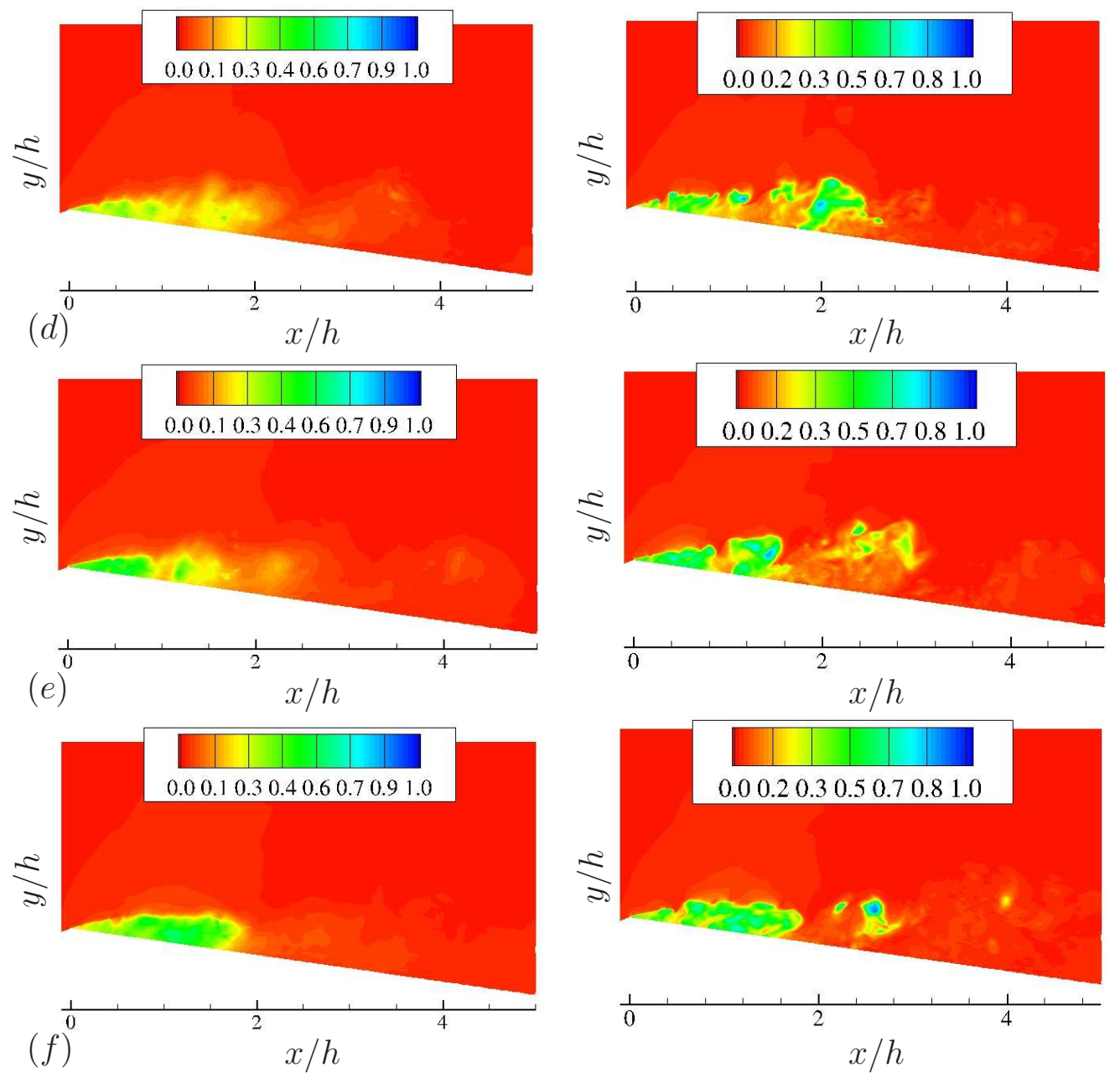

Figure 15: Time evolution of sheet to cloud transition. (Left) Instantaneous span-averaged void fraction contours, (Right) Instantaneous void fraction contours in the symmetry plane. 


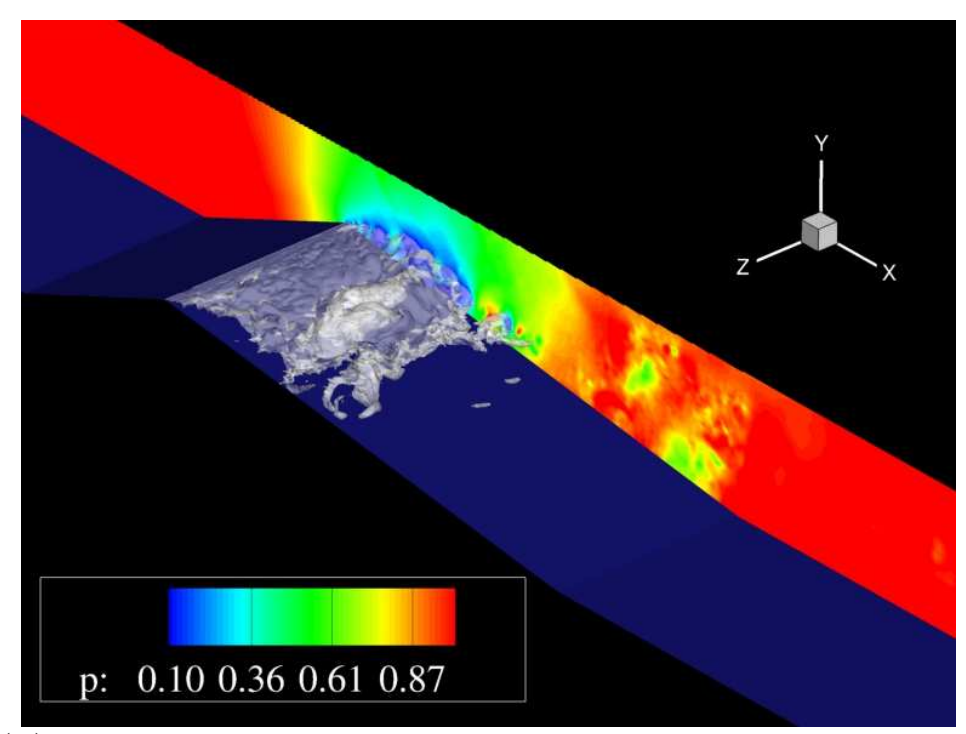

(a)

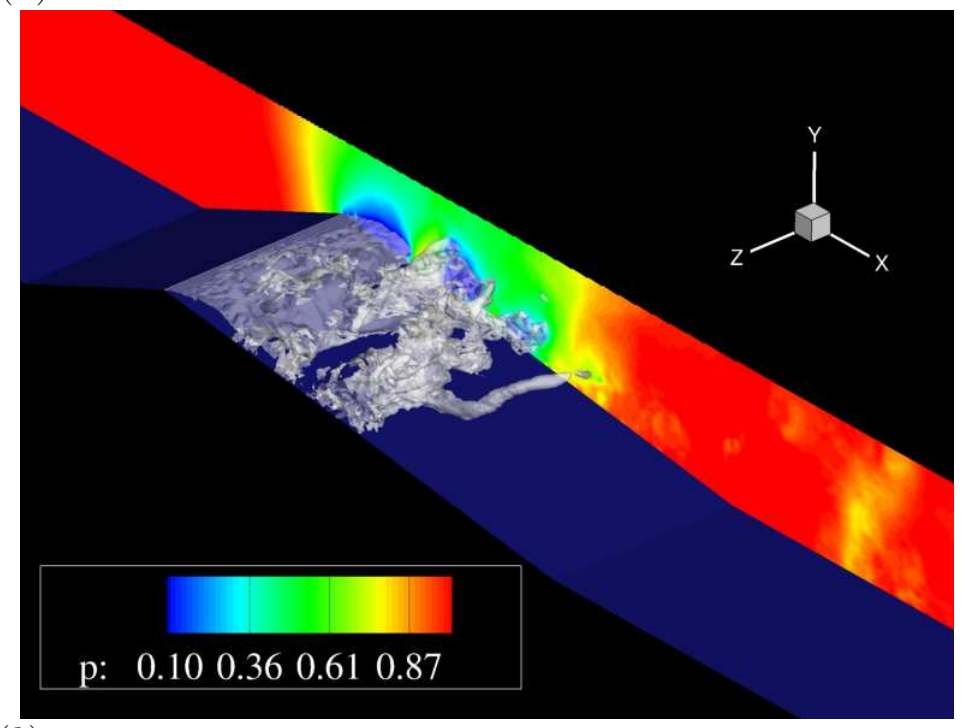

(b)

Figure 16: See next page for caption.

presence of multiple small cavities. These multiple smaller cavities get represented as two secondary clouds in the span-averaged contours. This three-dimensional feature is often not captured in $2 D$ unsteady RANS simulation. The sheet cavity then grows to its maximum length and the entire cycle repeats again. Figure 16 shows the isocontours of void fraction showing the three-dimensional view of the sheet to cloud transition process. The fully grown sheet cavity is evident in (a) and a clear detached cloud is seen in (b). The secondary shedding and the three-dimensional nature of the secondary cloud is evident in (c) and finally (d) shows the sheet cavity close to it full length before the next cycle begins. The side plane in Figure 16 shows pressure contours. Low pressure signatures seen downstream in (a) correspond to the cloud that had shed and collapsed from the previous cycle. Similar low pressure signatures can also be observed in (c) and (d).

The frequency of the shedding phenomenon (both primary and secondary) can be estimated from the point spectra computed from the time history of pressure at several locations inside the cavity and in the wake of the cavity. Figure 17 shows the time history of pressure and its corresponding spectrum in the frequency domain at three 

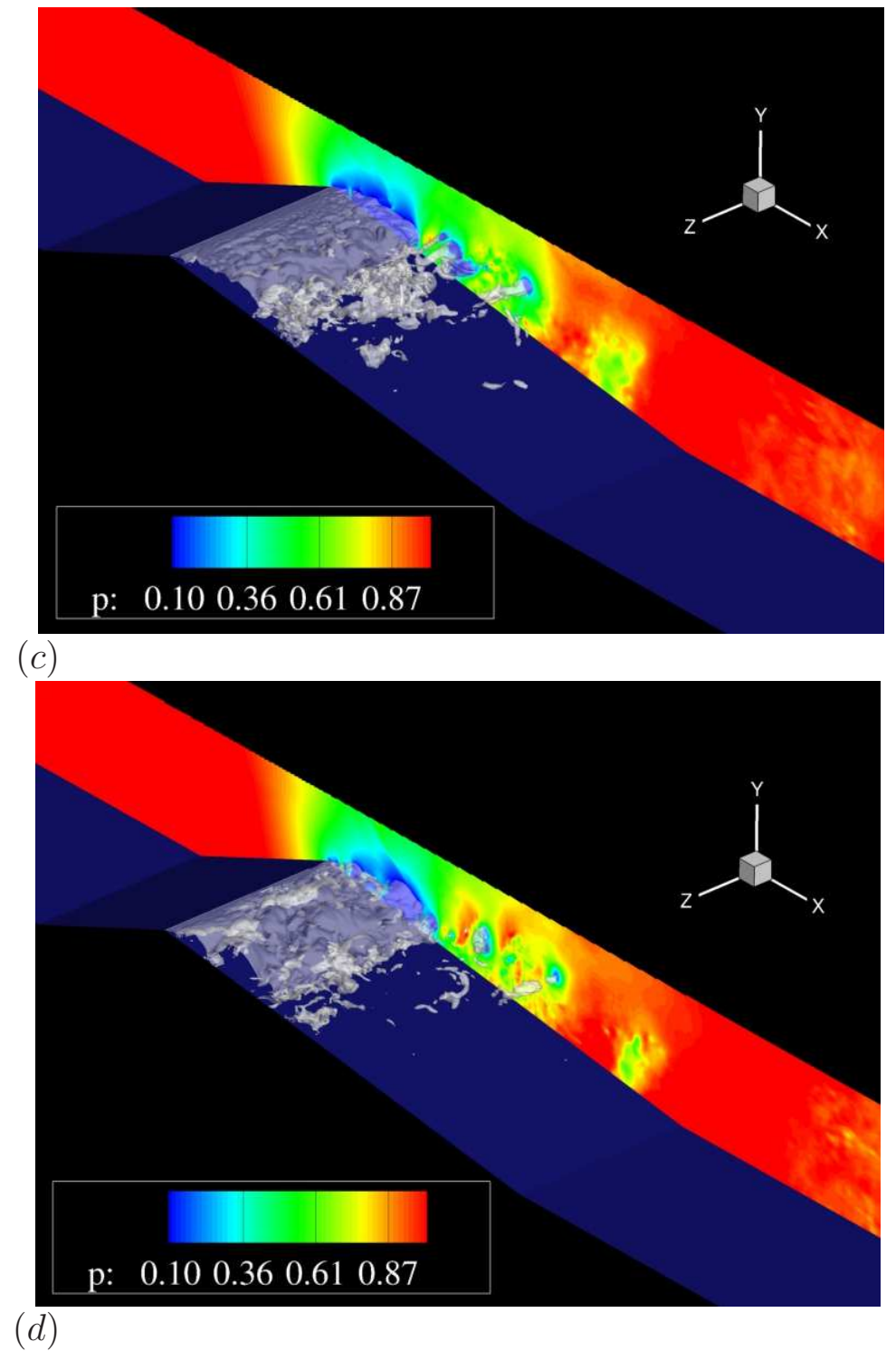

Figure 16: Three dimensional isocontours of void fraction $(\alpha=0.15)$ showing time evolution of sheet to cloud transition. Side plane shows contours of pressure. 
different locations $x / h=0.1,2.5$ and 4.5 and adjacent to the wedge wall. The spectra at $x / h=2.5$ and 4.5 show a peak at $S t=0.14$, where $S t=f h / u_{\infty}$. The shedding Strouhal number however is always expressed using maximum cavity length and hence $S t_{\text {shedding }}=f l_{\text {cav }} / u_{\infty}$. Given that the cavity grows to a maximum length $l_{\text {cav }} / h=2.0$, the shedding Strouhal number is 0.28. This value lies within the range of $0.25-0.3$ predicted by the experiment [13]. The spectrum at $x / h=0.1$ shows a maximum peak at $S t=0.3$. This peak corresponds to the secondary shedding which occurs at a higher frequency compared to the primary shedding i.e. many smaller clouds shed within a cycle at a higher frequency. The time history of pressure at $x / h=0.1$ also clearly indicates this trend, with pressure peaks occurring at a frequency corresponding to $S t=0.3$.

\subsection{Re-entering jet}

The importance of adverse pressure gradient in the cavity closure region in the development of re-entering jet has been studied extensively by Laberteaux and Ceccio [26] and Callenaere et al. [5]. In Figure 18(a), the time history of non-dimensional pressure immediately downstream of the mean cavity closure $(x / h=2.5)$ is plotted. Also shown in Figure 18(b) is the time history of streamwise velocity at $x / h=0.5$ and adjacent to the wall. A clear correlation is observed between the pressure peaks and negative velocity peaks. This implies that a strong re-entering jet is formed due to the adverse pressure gradient. Figure 18(c) shows the pressure evolution at a downstream location, through which the cloud cavity is expected to advect. The sudden drop in pressure observed in 18(c) is indeed due to the cloud passing through and it can be observed that these drops in pressure also correlate with peaks in re-entering jet. The main cloud shedding events are marked as $x$ and $z$ respectively and one of the secondary cloud shedding event is marked as $y$. For visualizing the re-entering jet, span averaged values are considered. Although spanwise variation in the re-entering jet is present, this method will give us details about the mean behavior. Streamlines plotted at three different time instants within a cycle are shown in Figure 19 and it shows the presence of a re-entering jet where a stream of liquid from the cavity closure enters into the cavity. The re-entrant jet then penetrates towards the apex of the wedge and the cavity pinches off close to the apex of the wedge.

\subsection{Pressure waves}

When a vapor cavity collapses, it creates a void and surrounding water rushes into the void creating a water hammer effect. This causes a large amount of pressure to be concentrated locally resulting in compression waves that travel in all directions. Due to the geometry confinement, these pressure waves get reflected multiple times causing a complex pressure wave pattern. These pressure waves also travel towards the inflow and modifies the oncoming flow significantly. Figure 20(a) shows the pressure history at three locations upstream of the wedge. The pressure increase caused due to pressure waves can be seen and it can be observed that the pressure at these locations are highly correlated. The direction of the pressure waves is towards the inlet. The spectra of pressure history at all these locations look identical as seen in Figure 20(b), with a dominant peak at $S t=0.16$. Although the pressure waves are caused due to cloud collapse, the complex interaction between the pressure waves among themselves and the domain boundaries mean that, the Strouhal number observed at the upstream locations is neither equal to the primary shedding Strouhal number nor the secondary shedding Strouhal number.

The pressure waves have both cyclic and intermittent behavior which can be observed from Figure 21 showing lines of pressure in the side plane. The highly intermittent 

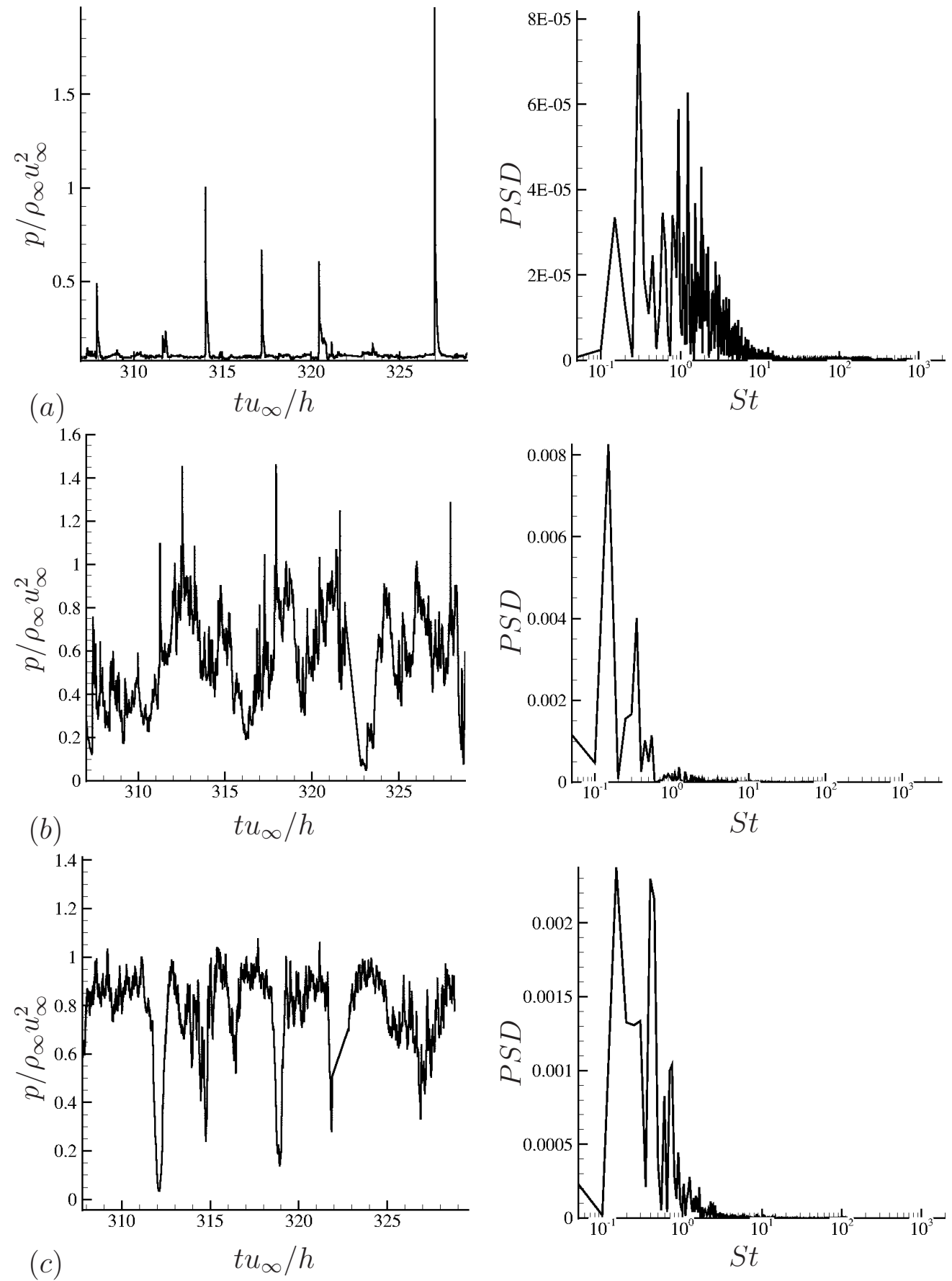

Figure 17: Time history of pressure (left) and their corresponding spectra (right), $(a): x / h=0.1,(b)$ : $x / h=2.5$ and $(c): x / h=4.5$. 

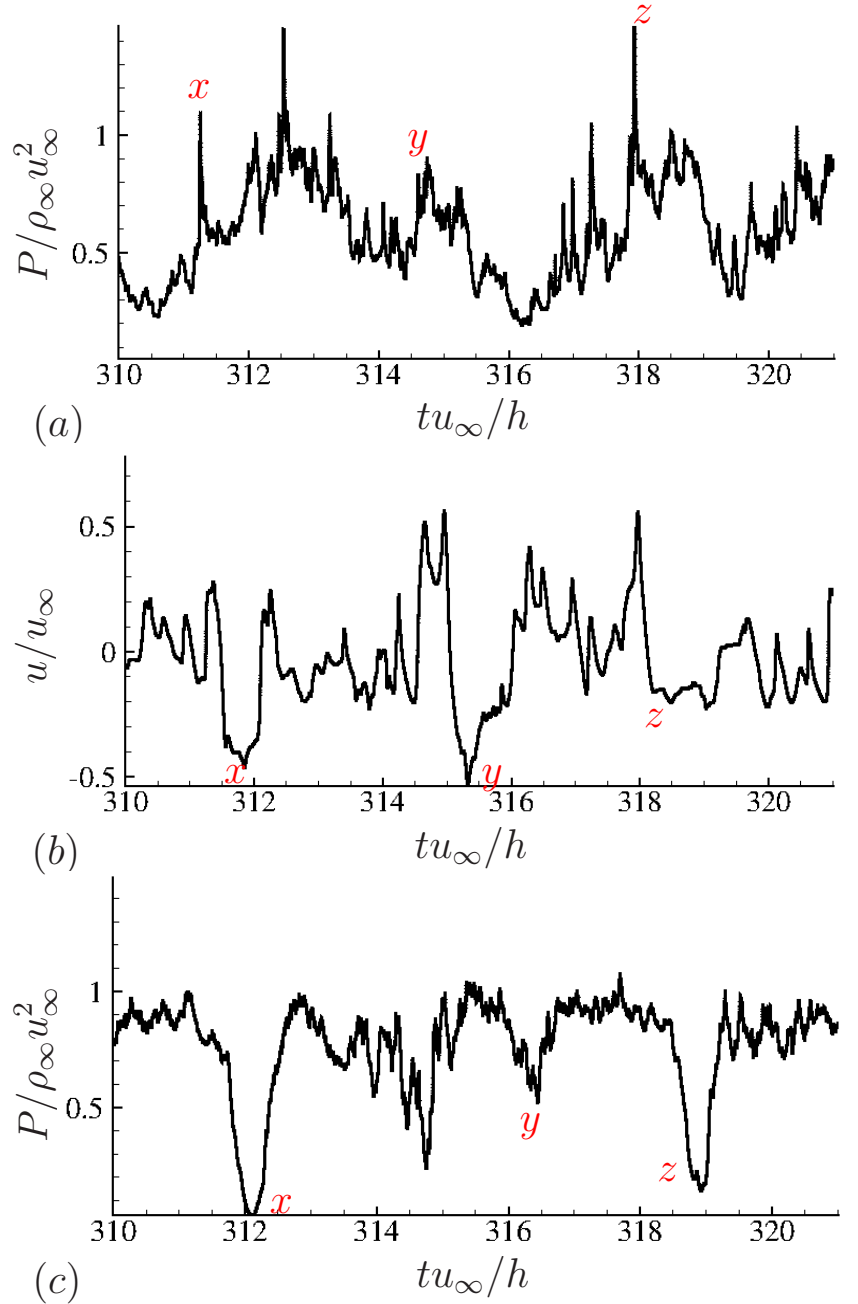

Figure 18: Time history of $(a)$ pressure at $x / h=2.5,(b) u$-velocity at $x / h=0.5$ and $(c)$ pressure at $x / h=4.5$. 

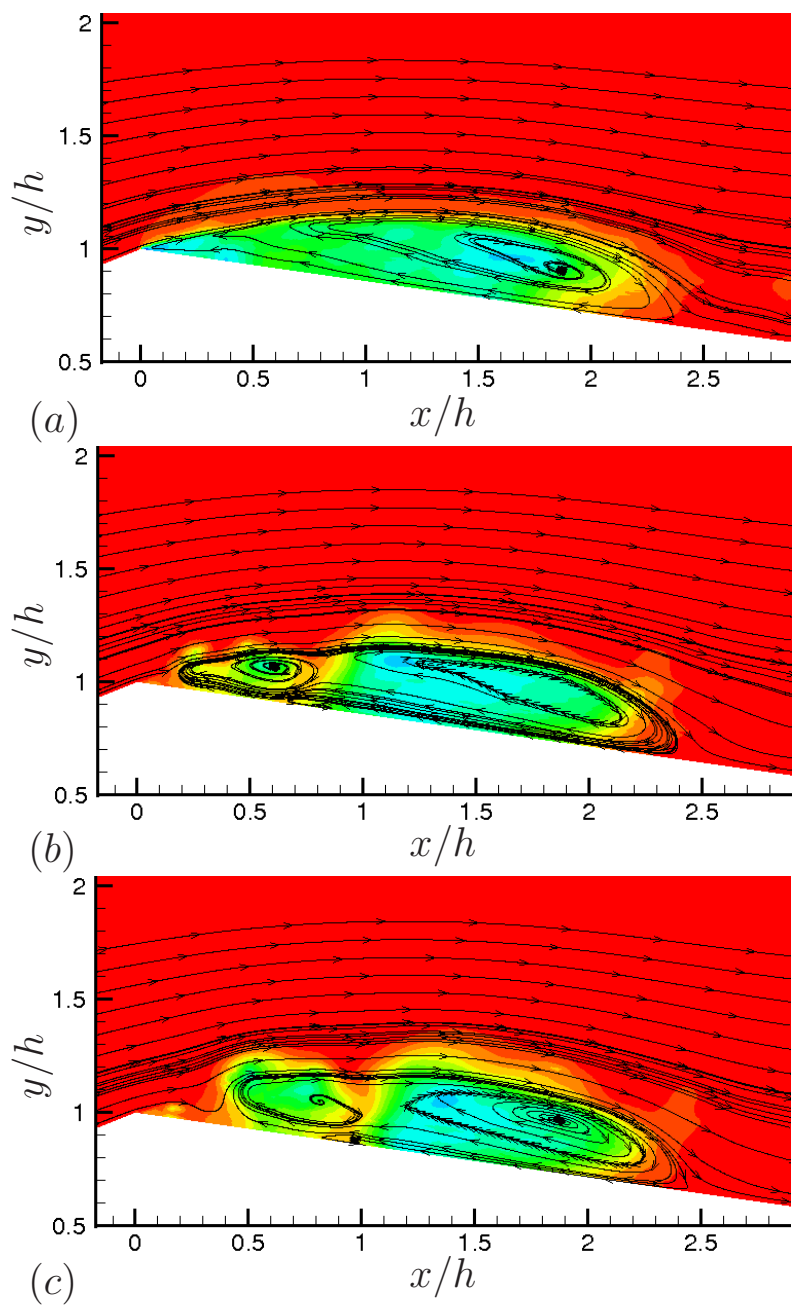

Figure 19: Three instants within a cycle showing the re-entering jet.

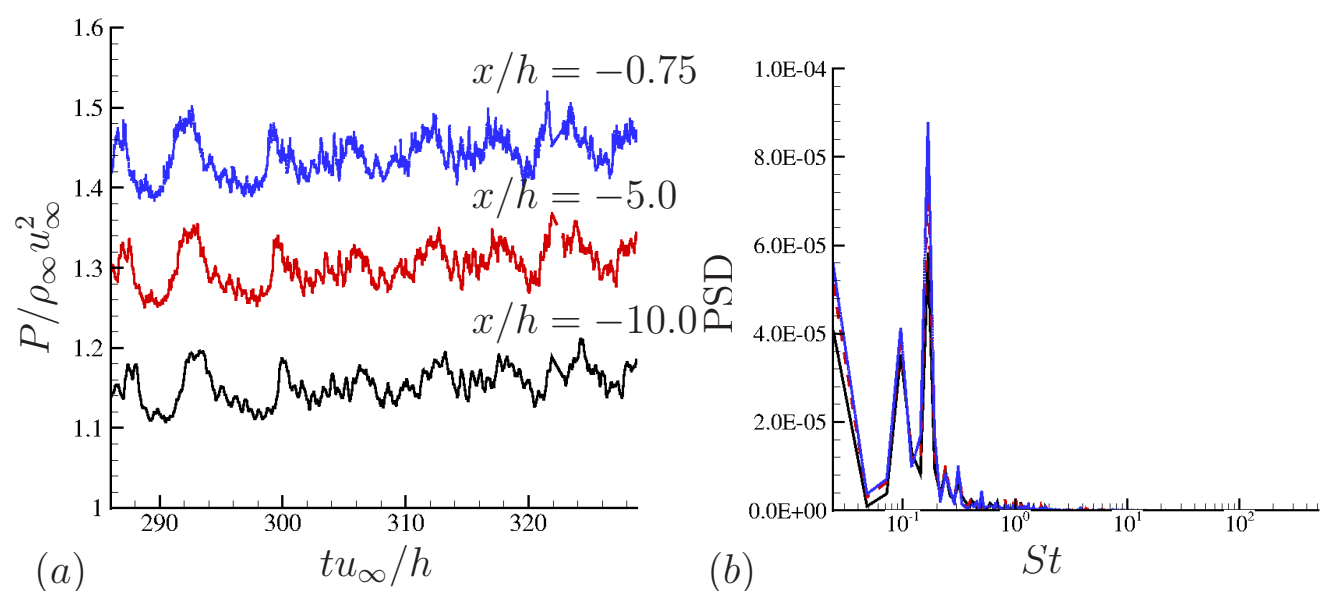

Figure 20: Pressure history upstream of the wedge showing the effect of pressure waves. 


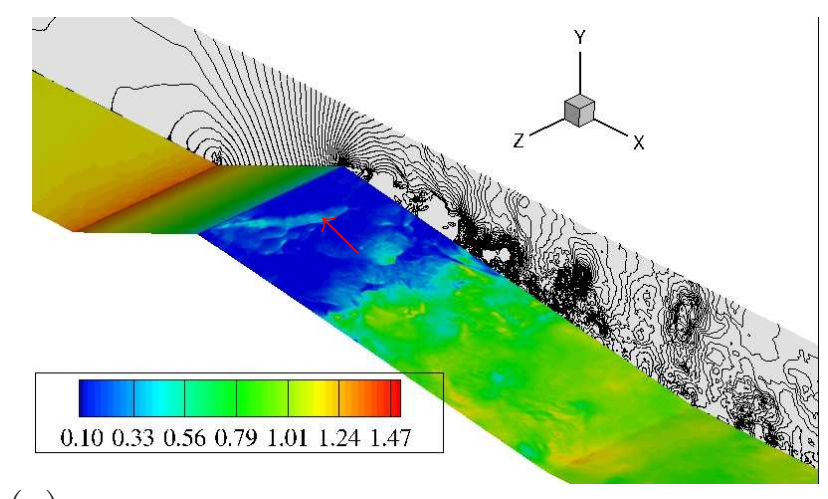

(a)

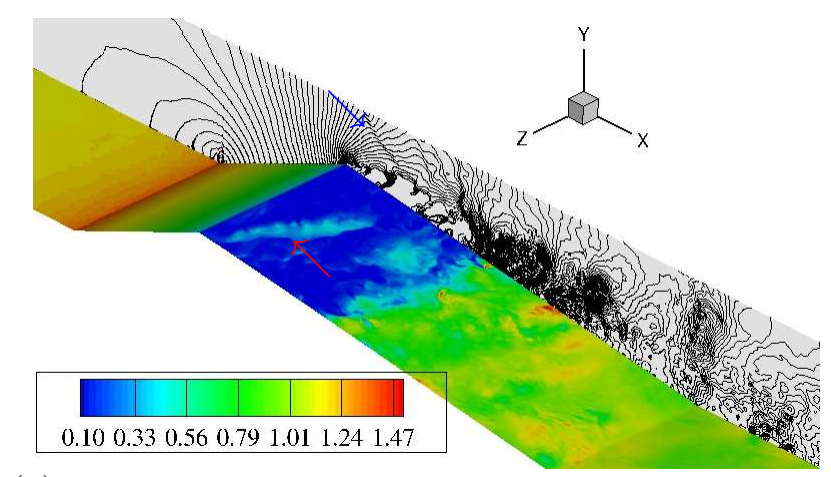

(b)

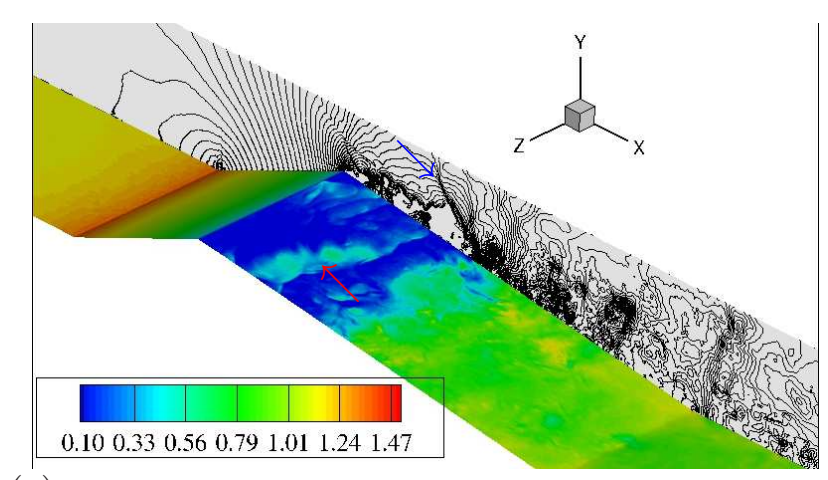

(c)
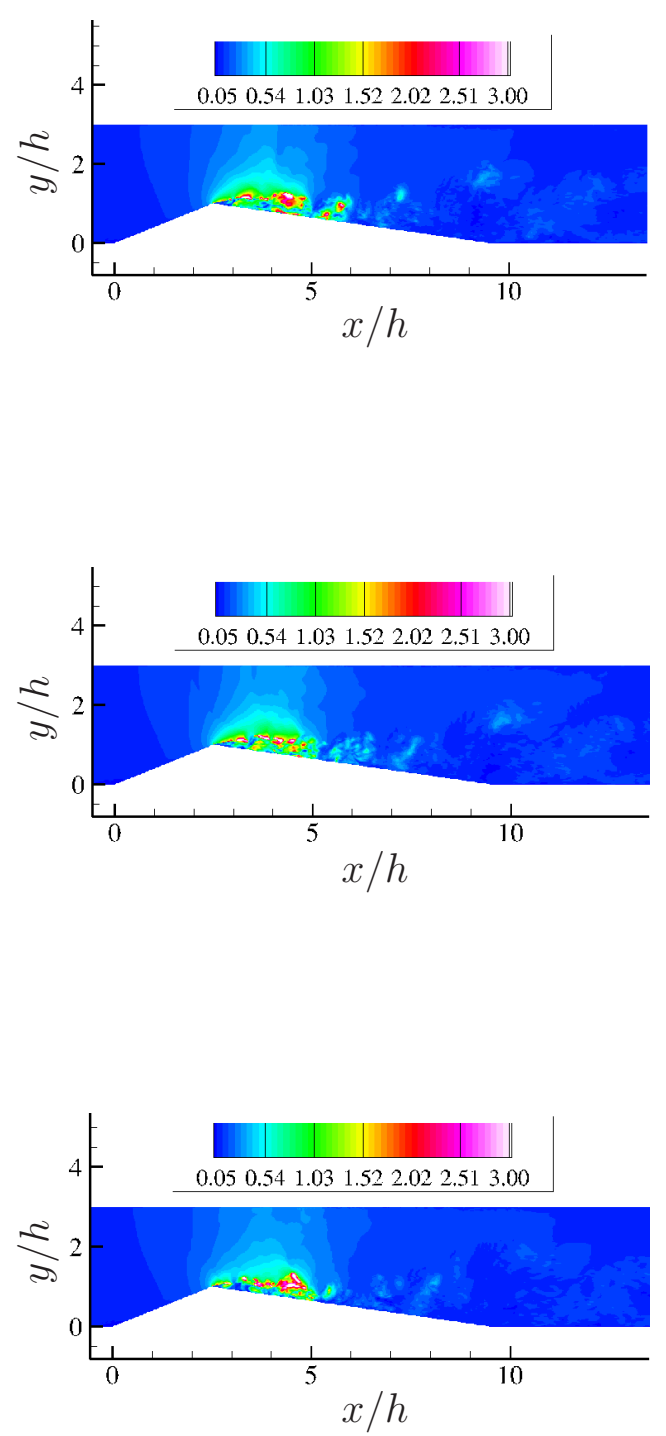

Figure 21: (Left) Pressure waves caused due to cavity collapse showing highly intermittent behavior. The bottom wall shows pressure contours and the side wall shows contour lines of pressure. (Right) Instantaneous Mach number contours in the symmetry plane. 
turbulent pressure fluctuations can be observed downstream of the cavity. The white region without pressure lines indicates the presence of the cavity. The pressure waves produced by collapse marked by a blue arrow mark are seen to impinge on the cavity. Pressure contours on the wedge wall also shed some light on the three-dimensionality of the vapor cavity. A pressure front across the span can be observed on the wedge wall in Figure 21 and the leading edge of the front is denoted by the red arrow mark. This indicates the front (cavity interface) separating the cloud from the sheet and not the pressure wave produced due to collapse. The process of cloud detachment is also observed to be three dimensional with variations across the span. The Mach number contours on the right show the presence of supersonic values in cavity region. The thickness of the supersonic regions is observed to be of the order of the cavity thickness. The interface of supersonic-subsonic regions could potentially indicate the presence of bubbly shock regions within the cavity.

\subsection{Vorticity production and modulation by cavitation}

The interaction of cavitation and vorticity can be understood by plotting the contribution of various terms in the vorticity equation,

$$
\frac{\partial \boldsymbol{\omega}}{\partial t}=-(\boldsymbol{v} \cdot \boldsymbol{\nabla}) \boldsymbol{\omega}+(\boldsymbol{\omega} \cdot \boldsymbol{\nabla}) \boldsymbol{v}-\boldsymbol{\omega}(\boldsymbol{\nabla} \cdot \boldsymbol{v})+\frac{1}{\rho^{2}}(\boldsymbol{\nabla} \rho \times \nabla p)+\boldsymbol{\nabla} \times \frac{\boldsymbol{\nabla} \cdot \tau}{\rho} .
$$

An important term in the right hand side of the vorticity equation that can lead to vorticity production is the baroclinic vorticity term. This arises due to the non-alignment of pressure and density gradients, which one would normally expect in the cavitated regions since pressure is not a function of density alone in such regions. Another important term, although not a production term, is vorticity dilatation which plays a major role in modulating vorticity. If a vortex undergoes compression, its vorticity is increased and if it undergoes expansion its vorticity is decreased. This is an important factor to consider since the core of vortices tend to cavitate more if the vorticity is increased. Figure 22 shows instantaneous span averaged contours of spanwise vorticity, baroclinic production of spanwise vorticity and vorticity dilatation of spanwise vorticity. Two time instants are considered; one during a fully formed cavity stage and the other during a cavity breakup stage. In both time instants, one can observe the dominant negative spanwise vorticity both in the form the unbroken shear layer on the sheet cavity and also in the small scale vortices downstream of the sheet cavity. As evident, vorticity is present in the cloud region even after the collapse. The thin layer of positive vorticity close to the wall is the vorticity associated with the re-entering jet.

Baroclinic vorticity is produced predominantly inside the sheet cavity, where maximum misalignment of pressure and density gradients are expected. In particular the mass transfer along the cavity surface ensures that baroclinic torque is maximum close to the shear layer. In fact, for the fully formed sheet cavity, one can observe lesser baroclinic torque inside the sheet cavity as compared to that close to the cavity surface (shear layer). The vorticity thus produced due to baroclinic torque and the shear layer is fed into the cloud that detaches from the sheet. Opposite signs of baroclinic vorticity very close to each other points to the highly three-dimensional turbulent nature of the flow. Inside the cloud region, both pressure gradient and density gradient are more or less in the same direction, hence lower magnitude of baroclinic vorticity.

The magnitude of vorticity dilatation is of the order of baroclinic production. In the sheet region, the predominant vorticity is in the clockwise direction; hence positive 

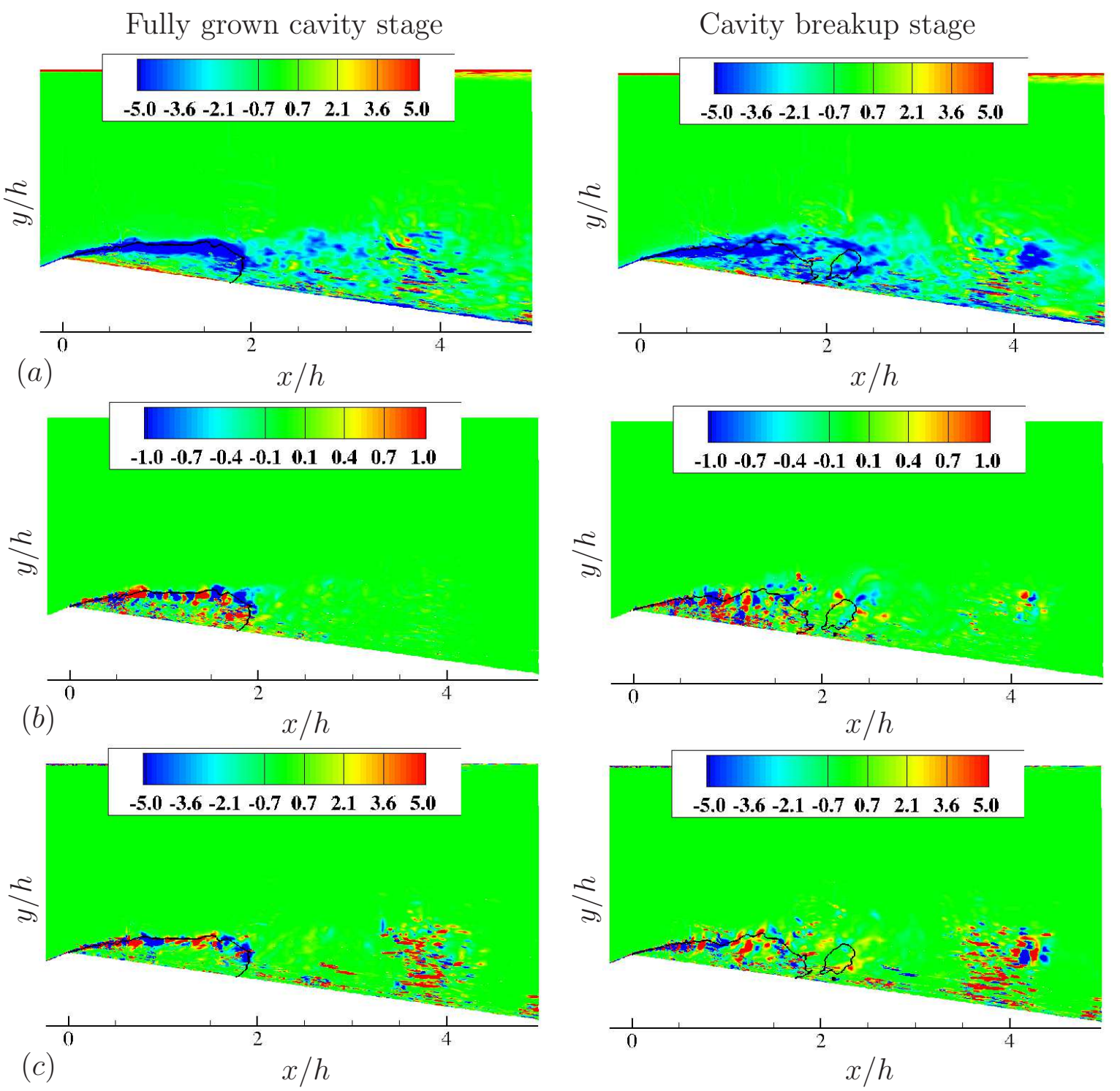

Figure 22: (a)Instantaneous span-averaged spanwise vorticity contours, (b) Instantaneous span-averaged baroclinic vorticity contours and $(c)$ Instantaneous span-averaged vorticity dilatation contours. (Left) Fully grown cavity stage and (Right) Cavity breakup stage.

vorticity dilatation contours are vorticity compression due to the smaller vapor cavities locally condensing back to liquid. The negative dilatation contours are vorticity expansion due to cavitation. In the cloud region, compression is dominant and hence vorticity dilatation leads to an increase in vorticity there.

\section{Summary}

Large Eddy Simulation is performed to study sheet to cloud cavitation transition over a wedge. The quantitative agreement of LES with experiment for this highly complex flow is encouraging. The Strouhal number corresponding to cloud shedding shows good agreement with the experiment. The study sheds light on the structure of the mean cavity. The velocity fluctuations indicate that only streamwise fluctuations are dominant inside the cavity, while both streamwise and spanwise fluctuations are equally dominant at the cavity closure and in the cavity wake. Both the sheet and cloud cavities are unsteady and highly three-dimensional. The anisotropy in the velocity fluctuations are higher in the cloud region as compared to the sheet region. The probability density function of void 
fraction plotted at several locations inside the cavity shows that the mean value obtained from time averaged data is very different from the most probable value of void fraction indicating the unsteadiness of the flow. The time evolution of void fraction shows that multiple smaller cavities are also shed apart from a main cloud. This highly unsteady phenomenon is often not captured in unsteady RANS simulations. These smaller cavities in the instantaneous snapshots look like two secondary cavities in the span-averaged contours. The point spectra at various points inside the cavity clearly shows the primary and secondary shedding frequency. The time history at these points also show a clear correlation between adverse pressure gradient, re-entering jet and the cloud formation. The cloud collapse results in pressure waves which have both cyclic and highly intermittent small scale behavior. The vorticity analysis shows the predominant role of baroclinic torque in producing vorticity and the role of compressibility in modulating the vorticity through the vorticity dilatation term.

\section{Acknowledgment}

This work was supported by the United States Office of Naval Research under ONR Grant N00014-11-1-0497 with Dr. Ki-Han Kim as technical monitor. Computing resources were provided by the Minnesota Supercomputing Institute (MSI), Texas Advanced Computing Center (TACC) and Arctic Region Supercomputing Center (ARSC)

\section{References}

[1] Ahuja, V., Hosangadi, A., and Arunajatesan, S. (2001). Simulations of cavitating flows using hybrid unstructured meshes. Journal of Fluids Engineering, 123(2):331-340.

[2] Arndt, R. E. A., Song, C. C. S., Kjeldsen, M., He, J., and Keller, A. (2000). Instability of partial cavitation: a numerical/experimental approach. In Proceedings of the 23rd Symposium on Naval Hydrodynamics, pages 519-615. National Academies Press.

[3] Arndt, R. E. A., Wosnik, M., and Qin, Q. (2006). Experimental and numerical investigation of large scale structures in cavitating wakes. In Proceedings of the 36th AIAA Fluid Dynamics Conference and Exhibit, AIAA Paper, number 3046, pages 1-8.

[4] Bensow, R. E. and Bark, G. (2010). Implicit LES predictions of the cavitating flow on a propeller. Journal of Fluids Engineering, 132(4):1-10.

[5] Callenaere, M., Franc, J.-P., Michel, J., and Riondet, M. (2001). The cavitation instability induced by the development of a re-entrant jet. Journal of Fluid Mechanics, 444:223-256.

[6] Colonius, T. (2004). Modeling artificial boundary conditions for compressible flow. Annual Review of Fluid Mechanics, 36:315-345.

[7] Coutier-Delgosha, O., Fortes-Patella, R., and Reboud, J.-L. (2003). Evaluation of the turbulence model influence on the numerical simulations of unsteady cavitation. Journal of Fluids Engineering, 125(1):38-45.

[8] Coutier-Delgosha, O., Stutz, B., Vabre, A., and Legoupil, S. (2007). Analysis of cavitating flow structure by experimental and numerical investigations. Journal of Fluid Mechanics, 578:171-222. 
[9] Dittakavi, N., Chunekar, A., and Frankel, S. (2010). Large eddy simulation of turbulent-cavitation interactions in a venturi nozzle. Journal of Fluids Engineering, 132(12):1-12.

[10] Edwards, J. R., Franklin, R. K., and Liou, M.-S. (2000). Low-diffusion flux-splitting methods for real fluid flows with phase transitions. AIAA journal, 38(9):1624-1633.

[11] Foeth, E., Terwisga, T. V., and Doorne, C. V. (2008). On the collapse structure of an attached cavity on a three-dimensional hydrofoil. Journal of Fluids Engineering, 130(7):071303.

[12] Frikha, S., Coutier-Delgosha, O., and Astolfi, J.-A. (2009). Influence of the cavitation model on the simulation of cloud cavitation on $2 \mathrm{D}$ foil section. International Journal of Rotating Machinery, 2008:1-12.

[13] Ganesh, H. (2015). Bubbly shock propagation as a cause of sheet to cloud transition of partial cavitation and stationary cavitation bubbles forming on a delta wing vortex. $\mathrm{PhD}$ thesis, University of Michigan.

[14] Gnanaskandan, A. (2015). Development of methodology for LES of turbulent cavitating flows. PhD thesis, University of Minnesota.

[15] Gnanaskandan, A. and Mahesh, K. (2015). A numerical method to simulate turbulent cavitating flows. International Journal of Multiphase Flow, 70:22-34.

[16] Goncalves, E. and Patella, R.-F. (2009). Numerical simulation of cavitating flows with homogeneous models. Computers \& Fluids, 38(9):1682-1696.

[17] Hoekstra, M., Terwisga, T. V., and Foeth, E. J. (2011). Smp11 workshop-case 1: Delftfoil. 2nd Int. Sym. Marine Propulsors, Hamburg, Germany.

[18] Huang, B., Zhao, Y., and Wang, G. (2014). Large eddy simulation of turbulent vortex-cavitation interactions in transient sheet/cloud cavitating flows. Computers $\&$ Fluids, 92:113-124.

[19] Ji, B., Luo, X., Wu, Y., Peng, X., and Duan, Y. (2013). Numerical analysis of unsteady cavitating turbulent flow and shedding horse-shoe vortex structure around a twisted hydrofoil. International Journal of Multiphase Flow, 51:33-43.

[20] Ji, B., Luo, X. W., Arndt, R. E. A., Peng, X., and Wu, Y. (2015). Large eddy simulation and theoretical investigations of the transient cavitating vortical flow structure around a NACA66 hydrofoil. International Journal of Multiphase Flow, 68:121-134.

[21] Kawanami, Y., Kato, H., Yamaguchi, H., Tanimura, M., and Tagaya, Y. (1997). Mechanism and control of cloud cavitation. Journal of Fluids Engineering, 119(4):788794.

[22] Kim, S. (2009). A numerical study of unsteady cavitation on a hydrofoil. In Proceedings of the 7th International Symposium on Cavitation, number 56, pages 1-13.

[23] Koop, A. and Hoeijmakers, H. (2009). Numerical simulation of unsteady threedimensional sheet cavitation. In Proceedings of the 7th International Symposium on Cavitation, number 26, pages 1-12. 
[24] Kubota, A., Kato, H., and Yamaguchi, H. (1992). A new modelling of cavitating flows: A numerical study of unsteady cavitation on a hydrofoil section. Journal of Fluid Mechanics, 240(1):59-96.

[25] Kunz, R. F., Boger, D. A., Stinebring, D. R., Chyczewski, T. S., Lindau, J. W., Gibeling, H. J., Venkateswaran, S., and Govindan, T. R. (2000). A preconditioned Navier-Stokes method for two-phase flows with application to cavitation prediction. Computers \&f Fluids, 29(8):849-875.

[26] Laberteaux, K. R. and Ceccio, S. L. (2001). Partial cavity flows. Part 1. Cavities forming on models without spanwise variation. Journal of Fluid Mechanics, 431:1-41.

[27] Leroux, J.-B., Astolfi, J. A., and Billard, J. Y. (2004). An experimental study of unsteady partial cavitation. Journal of Fluids Engineering, 126(1):94-101.

[28] Park, N. and Mahesh, K. (2007). Numerical and modeling issues in LES of compressible turbulence on unstructured grids. In Proceedings of the 45th AIAA Aerospace Sciences Meeting and Exhibit, AIAA Paper, number 0722, pages 1-18.

[29] Pham, T. M., Larrarte, F., and Fruman, D. H. (1999). Investigation of unsteady sheet cavitation and cloud cavitation mechanisms. Journal of Fluids Engineering, 121(2):289296.

[30] Saito, Y., Takami, R., Nakamori, I., and Ikohagi, T. (2007). Numerical analysis of unsteady behavior of cloud cavitation around a NACA0015 foil. Computational Mechanics, 40(1):85-96.

[31] Schnerr, G. H., Sezal, I. H., and Schmidt, S. J. (2008). Numerical investigation of three-dimensional cloud cavitation with special emphasis on collapse induced shock dynamics. Physics of Fluids, 20(4):1-9.

[32] Senocak, I. and Shyy, W. (2002). A pressure-based method for turbulent cavitating flow computations. Journal of Computational Physics, 176(2):363-383.

[33] Senocak, I. and Shyy, W. (2004). Interfacial dynamics-based modelling of turbulent cavitating flows, Part-1: Model development and steady-state computations. International Journal for Numerical Methods in Fluids, 44(9):975-995.

[34] Seo, J. H. and Lele, S. (2009). Numerical investigation of cloud cavitation and cavitation noise on a hydrofoil section. In 7th International Symposium on Cavitation, number 0062, pages $1-15$.

[35] Singhal, A. K., Athavale, M. M., Li, H., and Jiang, Y. (2002). Mathematical basis and validation of the full cavitation model. Journal of Fluids Engineering, 124(3):617-624.

[36] Spalart, P. R. and Allmaras, S. R. (1992). A one equation turbulence model for aerodynamic flows. AIAA Journal, 94(439):1-22.

[37] Stutz, B. and Reboud, J.-L. (1997). Two-phase flow structure of sheet cavitation. Physics of Fluids, 9(12):3678-3686.

[38] Wang, G. and Ostoja-Starzewski, M. (2007). Large eddy simulation of a sheet/cloud cavitation on a NACA0015 hydrofoil. Applied Mathematical Modelling, 31(3):417-447. 
[39] Yee, H. C., Sandham, N. D., and Djomehri, M. J. (1999). Low-dissipative high-order shock-capturing methods using characteristic-based filters. Journal of Computational Physics, 150(1):199-238.

[40] Yu, X., Huang, C., Du, T., Liao, L., Wu, X., Zheng, Z., and Wang, Y. (2014). Study of characteristics of cloud cavity around axisymmetric projectile by large eddy simulation. Journal of Fluids Engineering, 136(5):1-10. 